\title{
Sand suspension and fluxes by wave groups and equivalent monochromatic waves
}

Joep van der Zanden ${ }^{1}$, Dominic A. van der A², Peter D. Thorne ${ }^{3}$, Tom O’Donoghue ${ }^{2}$, Jan S. Ribberink ${ }^{1}$

$\underline{\text { Revised manuscript submitted to Continental Shelf Research (January 2019) }}$

1. Water Engineering and Management, University of Twente, P.O. Box 217, 7500 AE, Enschede, the Netherlands

2. School of Engineering, University of Aberdeen, King's College, AB24 3UE, Aberdeen, United Kingdom

3. National Oceanographic Centre, 6 Brownlow Street, L3 5DA, Liverpool, United Kingdom

Keywords: suspended sand; sediment transport; wave groups; wave flume experiment; sand ripples; acoustic backscatter sensor.

\section{Abstract}

Sand transport dynamics under non-breaking regular waves and wave groups are studied in a large-scale wave flume. The water surface elevation, flow velocity, suspended sand concentration (SSC) distributions, and bedforms were measured over a horizontal test section of medium sand. The experiment involved five wave groups that had equal root-mean-square water surface elevation and mean period, but which differed in terms of modulation, wave group length, and short wave sequencing. In addition, three monochromatic wave conditions were generated which were equivalent to the wave groups in terms of root-mean-square water surface elevation or maximum wave height. Temporal and vertical distributions of SSC suggest a more effective sediment pumping for symmetric and backward leaning 'waxing' wave groups. Vertical distributions of the SSC phase lag indicate a more rapid vertical pumping for the shortduration wave group. Overall, the differences between wave group conditions are small and the time-averaged SSC and sediment diffusivity profiles are very similar. The total net suspended sand flux consists of current-related, long-wave-related and short-wave-related contributions that are all of similar magnitude. The net total suspended sand flux was always offshoredirected, but its magnitude depends on the vertical and temporal variation of SSC at short- and long-wave time scales. The results are used to discuss the applicability of the 'equivalent wave concept' for time-averaged SSC distributions and net suspended sand transport. 


\section{Introduction}

Coastal regions are of vital importance as they are commonly densely populated and offer various ecosystem services. Coastal systems are dynamic: their morphology changes in time as sediment is displaced by currents, waves, and turbulent velocities. A profound understanding of the morphodynamic processes at various time scales is relevant in view of protecting and reinforcing coastal systems. Despite ongoing efforts, the mechanisms explaining the wave-driven cross-shore transport of sediment are not fully understood, leading to uncertainties in numerical morphodynamic models that are applied for research and engineering purposes.

As indicated by van Rijn et al. (2013), a specific topic that requires further research is the cross-shore transport of sand under irregular and grouped waves. Waves propagating toward a sandy beach exert a shear stress on the bed which entrains sand grains. Due to the dynamic flow fields under individual waves and under wave groups, the suspended sand concentration (SSC) varies at both short-wave and long-wave (infragravity-wave) frequencies (Hanes \& Huntley, 1986; Beach \& Sternberg, 1988; Osborne \& Greenwood, 1992). Depending on the temporal variation in SSC compared to the wave-induced orbital velocities, the short and long waves may transport suspended sand in the onshore or offshore direction (Jaffe et al., 1984; Osborne \& Greenwood, 1992; Ruessink et al., 1998).

Shoaled waves, which have propagated to intermediate and shallow waters, are generally positively skewed: the wave crests are high and of short duration relative to the shallow, long-duration wave troughs. Skewed waves induce different sand pick-up and vertical mixing rates between the crest and trough phase, which leads to a phase-dependent variation in SSC and to a net wave-related cross-shore sand flux. The magnitude and direction of this net flux depends on wave conditions, sand characteristics, and the presence of bedforms. For medium sand over flat beds, i.e. for high orbital velocities and bedload transport in a sheet flow regime, sand grains are picked up primarily during maximum bed shear stress by the orbital flow and, consequently, the SSC close to the bed is approximately in phase with freestream velocity (Ribberink \& Al-Salem, 1995). On the other hand, over steep sand ripples, the pick-up occurs predominantly during flow reversals when sediment-laden vortices are ejected into the flow (Sleath, 1982; van der Werf et al., 2007; O'Hara Murray et al., 2011; Finn et al., 2016). 
For positively skewed waves, the pick-up over a rippled bed is highest during the onshore-to-offshore flow reversal, commonly leading to maximum suspended load during the trough phase and a net offshore-directed wave-related suspended transport (Sato, 1987; Ribberink \& Al-Salem, 1994; van der Werf et al., 2006). The strongest time-dependency in SSC occurs in a near-bed layer of about two ripple heights that is characterised by convective mixing due to ripple-induced coherent vortices; at higher elevations these vortex structures are weakened and lead to more random diffusive mixing and a reduced time variation in SSC (Davies \& Villaret, 1997; Thorne et al., 2002; Davies \& Thorne, 2016). With distance from the bed, SSC exhibits a progressively increasing phase lag with respect to velocity and consequently, net wave-related sand fluxes may show strong vertical variation in terms of magnitude and direction (van der Werf et al., 2007; Hurther \& Thorne, 2011).

Sand suspension also responds to hydrodynamic forcing at the wave group time scale. Field measurements have shown that the SSC under short waves may be enhanced by the wave group (Hanes, 1991; Williams et al., 2002), which relates to a progressively increasing suspended sand load under the sequentially larger waves within a wave group (Osborne \& Greenwood, 1993; Hay \& Bowen, 1994), also referred to as 'sediment pumping' (Villard et al., 2000). Sediment pumping has been studied in more detail through wave flume experiments involving random waves (Villard et al., 2000; Vincent \& Hanes, 2002; Dohmen-Janssen \& Hanes, 2005; Williams \& Bell, 2006; O'Hara Murray et al., 2012) and bichromatic waves (Sato, 1992). With increasing distance from the bed, the SSC variations at the wave group time scale become progressively more significant compared to variations at short wave time scales (Dohmen-Janssen \& Hanes, 2005; O'Hara Murray et al., 2012). The SSC variations at wave group time scale lag the wave group envelope by about one short-wave half-cycle near the bed, but this phase lag increases progressively with distance from the bed, reaching values up to about 2-3 short-wave periods at elevations outside the wave bottom boundary layer (Villard et al., 2000; O'Hara Murray et al., 2012). The pumping process has been explained by a build-up of turbulent kinetic energy by ripple vortex shedding during a wave group which reduces the settling and promotes vertical mixing of suspended sediment (Villard et al., 2000; Vincent \& Hanes, 2002); numerical simulations of sand suspension over a flat bed reaffirm the important role of turbulence in sediment pumping (Holmedal et al., 2004). 
Suspended sand transport at long-wave (infra-gravity) frequencies generally tends to be offshoredirected under non-breaking wave groups (e.g. Ruessink et al., 1998; Vincent et al., 1999; Conley \& Beach, 2003; de Bakker et al., 2016). This can be explained by the fact that the larger waves in a group entrain the most sand, i.e., the suspended load is in phase with the wave group envelope, while the crossshore velocity associated with the bound long wave is in anti-phase with the envelope, thereby leading to a net offshore-directed long-wave transport (Shi \& Larsen, 1984; Sato, 1992; Deigaard et al., 1999). Since the magnitude of the long-wave transport depends on the phase coupling between SSC and the wave group envelope, insights into sediment pumping mechanisms contribute to an improved understanding of wave group effects on beach morphology.

Measurements by O'Hara Murray et al. (2012) suggest that wave group characteristics such as shape and duration could significantly affect the SSC dynamics. Except for the small-scale study by Sato (1992), the aforementioned studies on suspension under wave groups involved (repeating sequences of) random waves. Consequently, these studies did not allow a systematic and quantitative investigation of how the key characteristics of wave groups affect SSC dynamics and net suspended transport rates. Therefore, the present study aims to extend insights into the effects of wave group shape and duration on suspended sand transport dynamics and transport. The research objective is firstly to inter-compare suspended sand transport processes and net fluxes for non-breaking wave groups with similar offshore wave statistics. A second research objective is to study the equivalence in suspended sand transport dynamics between the wave groups and monochromatic waves.

The research approach consists of large-scale wave flume experiments involving non-breaking waves that propagate over a horizontal, medium-sand test section. The measurements and analyses focus on bedform dimensions, spatial and temporal SSC distributions, and net suspended sand fluxes. These features are studied for five bichromatic wave group conditions, with similar wave group energy and short-wave period, and three 'equivalent' monochromatic wave conditions.

The text is organised as follows. Section 2 describes the experiment and the data treatment. Section 3 presents the measured hydrodynamics, followed by an analysis of the measured bedforms in Section 4 . 
115 Section 5 presents the suspended sand concentration and sand flux measurements. The results are 116 discussed in Section 6 and Section 7 presents the main conclusions of this study.

\section{Methodology}

\subsection{Experimental description}

120

122

The experiments were conducted in the $100 \mathrm{~m}$ long, $3 \mathrm{~m}$ wide and $5 \mathrm{~m}$ deep CIEM wave flume at the Universitat Politècnica de Catalunya in Barcelona. Figure 1 illustrates the experimental set-up. The water depth $h_{0}$ near the wedge-type wave paddle was $2.65 \mathrm{~m}$. The experiment involved a mobile bed section consisting of medium sand (median diameter $D_{50}=0.24 \mathrm{~mm} ; D_{10}=0.15 \mathrm{~mm}, D_{90}=0.37 \mathrm{~mm}$; $D_{90} / D_{10}=2.5$ ) with a measured mean settling velocity $w_{\mathrm{s}}=0.034 \mathrm{~m} / \mathrm{s}$. Based on the measured geometric mean of moments $\left(\sigma_{\mathrm{g}}=1.36\right)$, the sand is classified as 'well sorted' (Blott and Pye, 2001). The initial bed profile consisted of a 1:10 offshore slope, followed by a $20 \mathrm{~m}$ long and $1.35 \mathrm{~m}$ high horizontal test section. The shoreward side of the test section was enclosed by a fixed beach, consisting of sand covered with geotextile and perforated concrete slabs that promoted wave energy dissipation. Throughout the paper, the horizontal coordinate $x$ is defined positively shoreward from the wave paddle in its lowest positon, the vertical coordinate $z$ is defined positively upward from the still water level, and the vertical coordinate $\zeta=z-z_{\text {bed }}$ is defined positively upward from the instantaneous local bed level.

\subsection{Measurements}

The water surface elevation $\eta$ was measured at 13 cross-shore locations using resistive wave gauges (RWGs) deployed from the flume side-walls (Figure 1). At eight additional locations, pressure transducers (PTs) were deployed from the flume side-walls, and another PT was deployed from the mobile frame. The water surface elevation was retrieved from these pressure measurements after correcting for pressure attenuation using linear wave theory (following Guza \& Thornton, 1980). All RWG and PT measurements were sampled at a data rate $f_{\mathrm{s}}=40 \mathrm{~Hz}$. 
Flow velocities, suspended sand concentration, and bedform geometry were measured with instruments deployed from a measurement frame that was attached to a carriage at the top of the flume. The frame could be vertically repositioned with sub-mm accuracy using a spindle, and was 'alongshore'-centred between the flume side-walls. More details on this 'mobile frame' can be found in Ribberink et al. (2014). The frame was positioned near the middle of the horizontal test section (at $x=58.5 \mathrm{~m}$ ). Figure 2 shows a photograph of the instrument arrangement on the frame.

Cross-shore and vertical velocities $u, w$ were recorded at $f_{\mathrm{s}}=100 \mathrm{~Hz}$ using a Nortek ADV-Vectrino, deployed from the mobile frame at free-stream elevation $\zeta=0.16 \mathrm{~m}$ and $x=58.4 \mathrm{~m}$. Vertical profiles of the time-dependent SSC were obtained with a multi-frequency AquaScat acoustic backscatter sensor (ABS). The ABS measured at acoustic frequencies of 2.0, 3.0 and 4.0 MHz, was deployed from the mobile frame at elevation $\zeta=0.82 \mathrm{~m}$ and cross-shore location $x=58.5 \mathrm{~m}$, and sampled with $5 \mathrm{~mm}$ vertical bin size resolution. The ABS measured the instantaneous backscattered signal at $f_{\mathrm{s}}=64 \mathrm{~Hz}$. The inversion from acoustic backscatter intensity profiles to SSC is explained in Section 2.5.

Additional measurements of the time-averaged SSC were obtained with a Transverse Suction System (TSS), consisting of seven stainless-steel nozzles, each connected through plastic tubing to a peristaltic pump on top of the flume. The nozzles had an intake diameter of $3 \mathrm{~mm}$ and an intake velocity of 2.3 $\mathrm{m} / \mathrm{s}$, which is approximately twice the maximum orbital velocity and therefore ensures a constant sediment trapping efficiency (c.f. Bosman et al., 1987). TSS sampling was started after one minute of wave action, when SSC reached a semi-equilibrium state (as confirmed by the ABS measurements). Suction samples were collected in 15 litre buckets, weighed and carefully drained to remove excess water, and then dry-weighed to determine the sand concentration. The obtained concentrations were corrected for the nozzles' trapping efficiency following Bosman et al. (1987). The uncertainty in TSS concentration measurements due to the processing procedures was estimated to be about $10 \%$. Variability in the vertical elevation of nozzles above the evolving bed introduces a further measurement uncertainty. The TSS design and procedures are explained in more detail in van der Zanden et al. (2017). The grain size distribution of each dry-weighed sample was measured with a laser-diffraction particle sizer (Beckman Coulter LS 13 320). 
Bedform geometry was measured with an acoustic sand ripple profiler (SRP), consisting of a $2 \mathrm{MHz}$

168

169

170

171

172

173

174

175

176

177

178

179

180

181

182

183

184

185

186

187

188

189

190

191

disc transducer mounted on a stepper motor, assembled in a waterproof aluminium and PVC housing (Bell \& Thorne, 1997b, 1997a; Bell et al., 1998). The transducer has a conical beam pattern of $1.1^{\circ}$ and the motor has a $0.9^{\circ}$ step size. The SRP was deployed from the mobile frame at $x=59.1 \mathrm{~m}$ and $\zeta=0.81$ $\mathrm{m}$. For the chosen settings, the SRP measured with a $0.6 \mathrm{~mm}$ along-beam bin resolution and over a $120^{\circ}$ swathe, corresponding to a bed level transect covering $1.8 \mathrm{~m}$ with horizontal resolution $\Delta x \approx 0.01 \mathrm{~m}$ in cross-shore direction and 0.6 to $1.2 \mathrm{~mm}$ in vertical direction. In continuous measuring mode, approximately three scans per minute were collected.

\subsection{Wave conditions}

Five wave group and three monochromatic wave conditions were generated. The wave group conditions comprised three bichromatic cases ('groups bichromatic', GB1, GB2 and GB3) and two sawtoothmodulated wave groups ('groups modulated', GM1 and GM2) as summarised in Table 1 and shown in Figure 3. Each wave group had the same root-mean-square (rms) water surface elevation $\eta_{\text {rms }}$ and mean short wave period $T_{\mathrm{m}}$.

The three bichromatic conditions (GB1, GB2 and GB3) were generated from two short wave components with frequencies $f_{1}, f_{2}$ and wave heights $H_{1}, H_{2}$ with a difference in frequency such that the group contained an integer number of half cycles. Table 1 presents the frequencies and wave heights of the bichromatic wave components. Conditions GB1 and GB2 are both fully modulated with $H_{1}=H_{2}=$ $0.30 \mathrm{~m}$, but condition GB2 is of shorter duration $\left(T_{\mathrm{gr}}=6.5 T_{\mathrm{m}}\right)$ than GB1 $\left(T_{\mathrm{gr}}=9.5 T_{\mathrm{m}}\right)$. Condition GB3 was generated from the same frequency components $f_{1}, f_{2}$ as condition GB1, but with different wave heights for each component $\left(H_{1} \neq H_{2}\right)$ resulting in a partially modulated group.

In addition, two ‘asymmetric groups' (GM1 and GM2) were generated by modulating a regular sine wave time-series (short wave period $T_{\mathrm{m}}=4.4 \mathrm{~s}$, wave height $H=0.30 \mathrm{~m}$ ) with a longer sawtooth-shaped wave signal that was based on Malarkey and Davies (2012), i.e.: 


$$
\eta(t)=A(t) \frac{H}{2} \sin \left(\frac{2 \pi t}{T_{\mathrm{m}}}\right)
$$

with

$$
A(t)=1+\frac{\arctan \left(\frac{b \sin \left(\frac{2 \pi t}{T_{\mathrm{gr}}}\right)}{1-b \cos \left(\frac{2 \pi t}{T_{\mathrm{gr}}}\right)}\right)}{\arctan \left(\frac{b}{\sqrt{1-b^{2}}}\right)}
$$

193

194 in which $T_{\mathrm{gr}}=44.0 \mathrm{~s}$ is the group period and $b=2 \beta-1$, where the factor $\beta$ describes the degree of 195 forward or backward leaning ('sawtoothiness'). A backward leaning 'waxing' group corresponds to $\beta<$ 0.5, while $\beta>0.5$ produces a forward leaning 'waning' group. For conditions GB1 and GB2, waxing and waning wave groups were produced by selecting $\beta=0.2$ and 0.8 , respectively (Figure 3d,e).

Experiments were also conducted for three monochromatic wave conditions (M1, M2, M3). These experiments were designed to be 'equivalent' to the corresponding grouped wave conditions at the horizontal test section (Table 1). All wave conditions were generated by the wedge-type wave paddle using steering signals based on first-order wave theory.

\section{$2.4 \quad$ Experimental procedure}

Each wave group experiment consisted of five consecutive 35-minute runs. Prior to each experiment, the flume was drained, bedforms were flattened out, and the bed profile shown in Figure 1 was restored. Table 1 indicates what was measured (SRP, ABS, TSS) during each run. Because the SRP interfered with the $2 \mathrm{MHz}$ ABS transducer, no simultaneous SRP and ABS measurements were collected. Single bedform scans with SRP were collected after each run; continuous scans were obtained during the $1^{\text {st }}$ and $3^{\text {rd }}$ run only for each condition. The continuous SRP data indicated that bedforms reached a quasiequilibrium state after about 15 minutes, which is well within the duration of the 35-min run. ABS 
measurements were therefore collected during every $2^{\text {nd }}, 4^{\text {th }}$ and $5^{\text {th }}$ run. TSS samples were collected during every $2^{\text {nd }}$ and $4^{\text {th }}$ run.

The monochromatic wave experiments also started from a restored bed profile. The duration of each monochromatic run was 22 min. Three runs were conducted for condition M1 and M2 and two runs for condition M3. Because of time constraints and because bed changes during these short runs were minor, the bed profile was not restored after each condition and the eight runs were instead conducted successively. SRP scans were collected after each run, and additional continuous scans were collected during the first run of conditions M1 and M2. The ABS measured during all other runs, but only the final run for each condition is used in the analysis to ensure that bedforms were adjusted to the wave condition. TSS samples were collected during the last run of each monochromatic condition.

\subsection{Data processing}

For the wave group conditions, the wave group envelope $\eta_{\text {env }}(t)$ was calculated based on the measured water surface elevations. The upper and lower envelopes ( $\left.\eta_{\text {env, upper }}(t), \eta_{\text {env, lower }}(t)\right)$ were calculated by cubic interpolation of the maximum and minimum water surface displacements for each short wave in the $\eta(t)$ time series. The envelope $\eta_{\text {env }}(t)$ was then calculated as $\eta_{\text {env }}(t)=\eta_{\text {env, upper }}(t)-\eta_{\text {env, lower }}(t)$.

Spurious ADV measurements were identified using minimum criteria for the signal-to-noise ratio criterion (15 dB) and correlation (50\%). Spurious velocity measurements were removed from the time series and were not replaced.

The radial-coordinate SRP backscatter was transformed to a regular grid using a triangular-based linear interpolation algorithm. Following O'Hara Murray et al. (2011), the bed level was defined as the level where the product of the backscatter and the gradient of the backscatter was maximum. The vertical accuracy of the SRP bed level measurements is approximately $5 \mathrm{~mm}$ (Thorne et al., 2002). Bedform dimensions were quantified by identifying the crests and troughs of each bedform. The bedform length

$235 \lambda_{\mathrm{r}}$ is defined as the cross-shore distance between two troughs. The bedform height $\Delta_{\mathrm{r}}$ is defined as the maximum crest height relative to the mean of the two adjacent troughs. 
The acoustic backscatter was converted to concentration $C$ using the implicit iterative routine described

238

239

240

241

242

243 by Thorne and Hurther (2014). The inversion routine was improved by accounting for vertical variations in median grain diameter based on the TSS measurements (Appendix A). The system constant $\mathfrak{R}$ of each ABS frequency transducer was calibrated using the TSS measurements of the time-averaged SSC. This calibration was done, per transducer, using all collected ABS and TSS data. For each transducer, a single value of $\Re$ was established and applied for the inversion of all runs. Similar to the SRP data, the bed level under each ABS transducer was obtained as the elevation where the product between backscatter and vertical gradient of the backscatter was maximum.

For the wave group conditions, the time series of water surface elevation, velocity, and SSC were decomposed into a low-frequency (long wave, annotated with subscript 'lf') and a high-frequency (short wave, annotated ' $h f$ ') component by applying an $8^{\text {th }}$-order Butterworth filter with 1/16 Hz cut-off frequency. This cut-off frequency was selected as it is approximately equal to $2 /\left(T_{\mathrm{m}}+T_{\mathrm{gr}}\right)$ for the shortest wave group (GB2), hence it correctly separates the short- and long-wave frequencies.

Time series of SSC profiles measured by ABS indicated that, for all conditions, it took about one minute before the suspended sand was vertically mixed over the $0.82 \mathrm{~m}$ depth. For this reason, data corresponding to the first minute were discarded. Phase-averaged quantities were then computed over the remainder of each run, corresponding to about 50 to 70 wave group repeats for the wave group conditions and about 250 repeats for the monochromatic wave conditions. Phase-averaged quantities are denoted with angular brackets and were computed, here for SSC, through:

$$
\langle C(\zeta, t)\rangle=\frac{1}{N} \sum_{n=0}^{N-1} C(\zeta, t+n T) \quad 0 \leq t<T
$$

where $T$ is equal to the wave period for the monochromatic waves and equal to the group period for the wave groups. All phase-averaged quantities are phase-referenced such that $t / T=0$ corresponds to the local beginning of the wave (group) cycle. Time-averaged quantities are denoted with an overbar. Periodic quantities are denoted with a tilde, e.g., the periodic or orbital velocity $\tilde{u}=\langle u\rangle-\bar{u}$. 
During each run, typically two to three sand ripples migrated below the measurement instrumentation.

262

263

264

265

266

267

268

269

270

271

272

273

274

275

Observations of the bed when the flume was drained revealed an irregular three-dimensional bed morphology. For this reason, the data were bed-averaged by transforming the vertical profiles of ABS concentration to local bed level coordinates (i.e., from $C(z)$ to $C(\zeta)$, with $\zeta=0$ corresponding to the instantaneous bed level) prior to phase-averaging. This means that the spatial variability of SSC over bedforms, as demonstrated by previous studies (Nakato et al., 1977; Fredsøe et al., 1999; Thorne et al., 2003; van der Werf et al., 2007; Hurther \& Thorne, 2011), is not considered in the present study. The irregularity of bedforms affects especially the time-dependent concentrations and net sand flux measurements at $\zeta / \Delta_{\mathrm{r}}<3$, which depend strongly on the phase of a migrating bedform (van der Werf et al., 2007; Hurther \& Thorne, 2011). The time-averaged suspended sand concentration, on the other hand, can be assumed to be well converged as its variation with bedform phase is minor (Hurther \& Thorne, 2011).

\section{Hydrodynamics}

The phase-averaged water surface elevation $\eta$ and free-stream horizontal velocity $u$ at cross-shore location $x=58.5 \mathrm{~m}$ are shown in Figure 4 (bichromatic groups GB1-3), Figure 5 (sawtooth-modulated groups GM1-2) and Figure 6 (monochromatic waves M1-3). Table 2 presents an overview of the main water surface and velocity parameters for all conditions. The velocity skewness $S k(\tilde{u})$ was calculated from the orbital velocity as follows

$$
S k(\tilde{u})=\overline{\tilde{u}(t)^{3}} /{\overline{\tilde{u}}(t)^{2}}^{3 / 2}
$$

\section{The orbital excursion diameter $d_{0}$ is commonly defined as the trajectory covered by a water parcel during} one wave half cycle, i.e., $d_{0}$ equals the time-integral of the orbital velocity $\tilde{u}$ over a half cycle. From the latter, it follows that the mean excursion diameter for irregular waves can be calculated through: 


$$
d_{0}=\frac{1}{2 N_{\mathrm{w}}} \int_{0}^{T_{(\mathrm{gr})}}|\langle\tilde{u}(t)\rangle| \mathrm{d} t
$$

with $N_{\mathrm{w}}$ is the number of short waves in the wave group ( $N_{\mathrm{w}}=1$ for monochromatic waves). In addition, the maximum orbital excursion diameter $d_{0, \max }$ was derived by time-integrating $\langle\tilde{u}\rangle$ over each wave halfcycle and taking the maximum.

All wave group conditions GB and GM were similar in terms of $\eta_{\mathrm{rms}}, \tilde{u}_{\mathrm{rms}}$, and $d_{0}$ (Table 2). The relatively small variability between conditions can be attributed to the differences in total wave energy dissipation between the wave paddle and the test section. For all wave group conditions, the time-averaged freestream velocity $\bar{u}$ is offshore-directed and is of small magnitude compared to the maximum onshore and offshore orbital velocity. At intra-group time scale the wave groups differ significantly, which can for instance be seen in the maximum and minimum water elevation, $\langle\eta\rangle_{\max },\langle\eta\rangle_{\min }$, and the maximum crossshore velocity in onshore and offshore direction, $\langle u\rangle_{\max },\langle u\rangle_{\min }$. From the GB conditions, the shortestduration bichromatic group GB2 contains the highest short wave and produces the flow with highest onshore velocity and highest velocity skewness (Figure 4, Table 2). The two GM conditions are similar in terms of $H_{\max },\langle u\rangle_{\max }$ and $\langle u\rangle_{\min }$ (Table 2), but the timing of the highest wave within the group is different (Figure 5). The sawtooth modulation further leads to higher $d_{0, \max }$ for the GM conditions relative to GB.

Note that the beach was too steep for complete dissipation of the incident short and long waves and that the wave paddle did not feature active wave absorption. In order to assess any effects of spurious incident and reflected long waves, the phase behaviour of the low-pass filtered water surface $\left\langle\eta_{\mathrm{lf}}\right\rangle$ and velocity $\left\langle u_{\mathrm{lf}}\right\rangle$ are examined (Figure 4 and Figure 5, dashed black lines). Figures 4a and 5a reveal that the trough of the long wave $\left\langle\eta_{\mathrm{lf}}\right\rangle$ occurs around $t / T_{\mathrm{gr}}=0.60$ to 0.75 , corresponding to a phase lag of $0.2 \pi$ to $0.5 \pi$ with respect to the short-wave envelope. This phase lag of the bound long wave is typical for shoaling wave groups and the magnitude of the observed phase lag is similar to previous observations (Janssen et al., 2003; Battjes et al., 2004; Padilla \& Alsina, 2017). The low-frequency velocity $\left\langle u_{\mathrm{lf}}\right\rangle$, on the other hand, is maximum in offshore direction under the highest short waves (Figures $4 \mathrm{~b}$ and $5 \mathrm{~b}$ ), consistent with equilibrium theory of Longuet-Higgins and Stewart (1962). Based on the agreement with previous 
studies, the effects of spurious incident and reflected free long waves on the hydrodynamics are expected to be minor and sand transport is considered to be largely controlled by the velocity forcing associated with the incident short and the bound long waves.

The measured water surface elevation and velocity for the monochromatic cases are shown in Figure 6 and the corresponding hydrodynamic parameters are summarised in Table 2. Conditions M1 to M3 are characterised by a progressively increasing wave height, which leads not only to an increase in $d_{0}$ and magnitudes of $\langle u\rangle_{\max }$ and $\langle u\rangle_{\min }$ but also to increasing return current magnitude and velocity skewness. The approximate equivalence with the corresponding grouped wave conditions follows from Table 2: condition M1 is equivalent to all GB and GM conditions in terms of $\eta_{\mathrm{rms}}$; M2 is equivalent to GB1 in terms of $H_{\max }$; M3 is equivalent to GB2, GM1, and GM2 in terms of $H_{\max }$. Note, however, that the equivalence in terms of velocity is limited. Most importantly, the monochromatic cases M2 and M3 have higher $\tilde{u}_{\text {rms }}$ and $S k(\tilde{u})$, and consequently, higher $d_{0}$ and $\langle u\rangle_{\max }$ than their GB and GM equivalents.

\section{Bedforms}

Sand suspension processes are strongly controlled by bedforms. Visual inspection of the bed after draining the flume revealed the formation of three-dimensional irregular sand ripples for all wave conditions. Table 3 presents the sand ripple dimensions which were extracted from the SRP measurements after each experimental run. Ripple migration speeds $c_{\mathrm{r}}$ were established from continuous SRP measurements for all conditions except M3. The relatively high standard deviations reflect the irregularity of the bedforms. For reference, Table 3 lists the non-dimensional velocity forcing parameters $d_{0} / D_{50}$ and $\psi_{\max }=\langle u\rangle_{\max }^{2} /\left((s-1) g D_{50}\right)$, where $s=2.65$ is the specific gravity of sand and $g$ is the gravitational acceleration.

The GB and GM conditions produce ripples with a height $\Delta_{\mathrm{r}} \approx 0.015 \mathrm{~m}$, length $\lambda_{\mathrm{r}} \approx 0.20 \mathrm{~m}$, and steepness $\Delta_{\mathrm{r}} / \lambda_{\mathrm{r}} \approx 0.07$. The differences in ripple dimensions between the various wave group conditions are small and within the measurement uncertainty. The monochromatic waves (M1-3) produce ripples that have a similar steepness but that are slightly higher and longer ( $\Delta_{\mathrm{r}} \approx 0.02$ to $0.03 \mathrm{~m} ; \lambda_{\mathrm{r}} \approx 0.25$ to $0.57 \mathrm{~m}$ ) 
compared to the wave group conditions. This likely relates to the higher flow excursion diameter $d_{0}$ for the monochromatic waves relative to the wave groups.

The non-dimensional bedform heights and lengths can be used to classify the ripples. Ripples can be classified as 'orbital' (when $\lambda_{\mathrm{r}}$ is proportional to $d_{0}$ ), 'suborbital' (when $\lambda_{\mathrm{r}}$ is inversely proportional to $d_{0}$ ), and 'anorbital' (when $\lambda_{\mathrm{r}}$ is independent of $d_{0}$ ) (Clifton \& Dingler, 1984). The increasing ripple size for condition M1 to M3 suggests that bedforms scale with $d_{0}$ and are therefore in an orbital ripple regime. Note that the widely applied classification by Wiberg and Harris (1994) would predict ripples in a suborbital range for the present flow and sediment conditions $\left(1800<d_{0} / D_{50}<5500\right)$. However, O'Donoghue et al. (2006) showed that the orbital regime may extend to $d_{0} / D_{50}>1800$ for full-scale laboratory experiments.

The ripple steepness has a large effect on suspended sand transport. For sufficiently steep ripples, flow separation will occur during each half-cycle, leading to the formation of sediment-laden vortices that contribute significantly to sand pick-up (Bagnold, 1946). This vortex ripple regime occurs for ripples with $\Delta_{\mathrm{r}} / \lambda_{\mathrm{r}}$ higher than 0.10 to 0.15 (Clifton \& Dingler, 1984; Malarkey \& Davies, 2002). The ripples in the present experiment (Table 3) do not exceed this threshold, on average. Such more gentle ripples have been termed 'post-vortex ripples’ (Clifton \& Dingler, 1984), and ‘dynamically plane’ beds (Davies \& Villaret, 2002); the term 'post-vortex ripples' is adopted in the present study. The vertical transfer of momentum and suspended sand grains over post-vortex ripples is due to turbulent diffusive processes rather than organised vortices (Davies \& Villaret, 2002; Amoudry et al., 2016) and the sand pickup rate is lower than over a vortex rippled bed (O'Hara Murray et al., 2011; Davies \& Thorne, 2016). Note that $12 \%$ of the measured irregular ripples have a steepness higher than 0.10 and could thus be classified as vortex ripples.

On the basis of measurements in oscillatory flow tunnels and wave flumes, O'Donoghue et al. (2006) presented the following empirical relations for ripple dimensions as function of mobility number $\psi$ :

$$
\frac{\Delta_{\mathrm{r}}}{d_{0}}=\frac{\alpha_{3 \mathrm{D}}}{2}\left(0.275-0.022 \psi^{0.42}\right)
$$




$$
\frac{\lambda_{\mathrm{r}}}{d_{0}}=\frac{\beta_{3 \mathrm{D}}}{2}\left(1.97-0.44 \psi^{0.21}\right)
$$

Here, the empirical constants $\alpha_{3 \mathrm{D}}=0.55$ and $\beta_{3 \mathrm{D}}=0.73$ account for the smaller dimensions of 3D ripples compared to 2D ripples (see, e.g., Sato et al., 1988; O’Donoghue et al., 2006). Following O'Donoghue et al. (2006), the mobility number $\psi$ is for regular waves based on the maximum velocity and for irregular waves on the mean of the highest one tenth peak velocity.

Figure 7 shows a quantitative comparison between the measured and predicted 3D ripple heights and lengths. The measured ripple heights and lengths correlate positively with $\psi$, which confirms that the ripples are in an orbital regime. The model and data agree reasonably well in terms of ripple height and length: the data corresponding to the wave groups and to condition M1 are within the $\pm 50 \%$ bands, which is the typical accuracy of the formula (see O’Donoghue et al., 2006). The monochromatic conditions M2 and M3 produce ripples that are higher and longer than predicted. O'Donoghue et al. (2006) indicated that their formula is applicable for $\psi \leq 190$ and that the model uncertainty is largest for 3D ripples at high mobility, which likely explains the discrepancy between measurements and predictions for conditions M2 and M3.

Bedform migration speeds $C_{\mathrm{r}}$ were quantified from the continuous bed level scans by comparing the start and end position of individual ripple crests for each run. Values of $c_{\mathrm{r}}$ are defined positively shoreward and are included in Table 3. Note that only few ripples could be tracked during an entire run, which explains the relatively high measurement uncertainty. It follows that ripples migrated onshore for all conditions, with slightly higher migration speeds for the regular waves than for the grouped waves.

\section{Suspended sand}

This section investigates the vertical and temporal distributions of suspended sand concentration (Sections 5.1 to 5.3), the net suspended flux distributions (Section 5.4) and net suspended sand transport rates. 


\subsection{Suspended sand concentration time series}

382

383

384

385

386

387

388

389

390

391

392

393

394

395

396

397

398

399

400

401

402

403

404

405

406

This section presents time series of the phase-averaged suspended sand concentration (SSC). The vertical mixing of suspended sand typically scales with the ripple height (Nielsen, 1984; van Rijn, 1993). In order to facilitate comparisons between the different conditions and with other studies, the vertical coordinate $\zeta$ is normalised by the mean ripple height $\Delta_{\mathrm{r}}$ for each condition.

Figure 8 shows the phase-averaged SSC for the monochromatic conditions. Figure 8a-c shows the concentration field as a colour contour; Figure 8d-f shows the concentration time series at $\zeta=\Delta_{\mathrm{r}}$, which can be expected to relate closely to the pick-up of sediment from the bed. For each condition, SSC decreases strongly with distance from the bed: from $C=10 \mathrm{~kg} / \mathrm{m}^{3}$ close to the bed $\left(\zeta / \Delta_{\mathrm{r}}<1\right)$ to $0.1 \mathrm{~kg} / \mathrm{m}^{3}$ at $\zeta / \Delta_{\mathrm{r}} \approx 5$. These magnitudes match closely to observations by Hurther and Thorne (2011) in the same experimental facility and for the same sediment. The concentrations at $\zeta=\Delta_{\mathrm{r}}$ differ notably between the conditions, with two pickup events for M1, three pickup events for M2 and one main pickup event for M3 (events indicated by arrows in Figure 8d-f). These differences are partly attributed to differences in bedform dimensions, as explained in what follows.

For condition M1 (Figure 8a), SSC between $\zeta / \Delta_{\mathrm{r}} \approx 1$ and 5 reveals two intra-wave peaks, corresponding to two main pickup events that are indicated by the arrows in Figure 8d. The highest pickup event occurs during crest-to-trough flow reversal at $t / T=0.35$. The entrained sediment spreads gradually upward to yield the main SSC peak at $\zeta / \Delta_{\mathrm{r}} \geq 3$ during the trough half-cycle $(t / T=0.80)$ (Figure 8a). A second, smaller, pickup event occurs during maximum negative velocity at $t / T=0.85$ (Figure $8 \mathrm{~d}$ ). The entrained sand associated with the trough-phase pickup, in combination with sand that has remained into suspension, is spread upward during the trough-to-crest flow reversal and produces the main suspension peak during the crest phase at $\zeta / \Delta_{\mathrm{r}} \geq 3$ (Figure 8a). The 'lifting' of the concentration field between $t / T=$ 0.9 and 0.2 in the next wave cycle is partly attributed to suspended sand advection by the upwarddirected orbital flow under the wave front.

Condition M2 induces three intra-wave suspension events (Figure 8e; $t / T=0.18,0.58$, and 0.85). Similar observations of three or more intra-wave suspension peaks were reported by van der Werf et al. (2007) 
over vortex ripples with similar $d_{0} / \lambda_{\mathrm{r}}$. Following the explanation by van der Werf et al. (2007), the suspension events at $t / T=0.18$ and 0.58 are associated with vortex formation around the negative-topositive and the positive-to-negative flow reversal, respectively, while the third suspension peak at $t / T$ $=0.85$ might relate to the horizontal advection of a sediment-laden vortex that was generated at the neighbouring ripple. Note that bedforms for conditions M1 and M2 were both qualified as post-vortex ripples, but ripple heights are considerably higher for M2 (Table 3). The latter may explain the qualitative agreement of the suspension events for condition M2 with observations over vortex ripples.

Condition M3 produces one main intra-wave pickup event that occurs shortly after the crest-to-trough flow reversal $(t / T=0.38)$, leading to maximum concentrations during the early stage of the trough half cycle (Figure 8c,f). The pickup during the trough half cycle is minor, although a small increase in $C$ can also be observed around the trough-to-crest half cycle $(t / T \approx 1.0)$. Note that for this condition, bedforms are longer and less steep than for conditions M1 and M2 (Table 3), hence reducing the tendency of flow separation and vortex-induced suspension to occur (e.g., Osborne and Vincent, 1996). The transport is likely confined to a very thin near-bed layer during most of the wave cycle and the threshold for suspension is only exceeded during the crest phase, when the velocity is maximum.

Comparison of SSC distributions for conditions M1 to M3 (Figure 8a-c) shows that the $C=0.1 \mathrm{~kg} / \mathrm{m}^{3}$ isoline progressively reaches higher intra-wave elevations with increasing wave height. This indicates that the vertical mixing rate at $\zeta / \Delta_{\mathrm{r}}>5$ increases with wave height. In contrast, the $C=1$ and $10 \mathrm{~kg} / \mathrm{m}^{3}$ isolines $\left(\zeta / \Delta_{\mathrm{r}}<5\right)$ are lower for M2 and M3 than for M1, which suggests a lower pickup rate. The latter could be attributed to the increasing bedform length, and the consequent reduction in $d_{0} / \lambda_{\text {r }}$, from condition M1 to M3 (Table 3): studies over vortex ripples have suggested a general reduction in vortex strength with decreasing $d_{0} / \lambda_{\mathrm{r}}$, with a lower limit of $d_{0} / \lambda_{\mathrm{r}}=1$ for flow separation and vortex suspension (Malarkey \& Davies, 2002; O'Hara Murray et al., 2011). Although most of the present ripples are in a post-vortex regime, the measurements indicate a similar trend of decreasing sand pick-up rates for decreasing $d_{0} / \lambda$. This may partly relate to the fraction (12\%, measured value) of ripples that have a steepness corresponding to a vortex ripple regime. 
Figure 9 shows the phase-averaged SSC for the grouped bichromatic (GB) waves. It is evident that SSC varies both at short-wave and wave-group time scales. As the suspended load gradually builds up during the wave group, the suspended load is much higher under short waves at the end of the group (e.g. $t / T_{\mathrm{gr}}$ $\approx 0.8)$ than under waves with similar wave height at the start of the group $\left(t / T_{\mathrm{gr}} \approx 0.2\right)$. This illustrates that SSC is not only controlled by the instantaneous wave forcing at short-wave time scale but is also strongly affected by the preceding waves. This 'sediment pumping' is consistent with other laboratory studies (Sato, 1992; Villard et al., 2000; Vincent \& Hanes, 2002; O'Hara Murray et al., 2012).

The three GB conditions in Figure 9 induce a similar response in SSC at short-wave time scales: the peaks in SSC due to short-duration 'clouds' of suspended sand near the bed $\left(\zeta / \Delta_{\mathrm{r}}<5\right)$ are approximately the same. However, at wave group time scale, condition GB1 shows a stronger build-up of the suspended sand load toward the end of the group ( $t / T_{\mathrm{gr}} \approx 0.8$ ) than conditions GB2 (shorter duration) and GB3 (only partially modulated). This higher build-up of the suspended load for GB2 relative to GB1 agrees with field observations by Williams et al. (2002), who also observed the suspended sand concentration to increase with the number of short waves in a group. On the other hand, at the start of the wave group $\left(t / T_{\mathrm{gr}} \approx 0-0.2\right)$ there is more sediment settling out of suspension for condition GB1 than for GB2 and GB3.

Figure 10 shows the phase-averaged SSC for the sawtooth-modulated GM conditions. Sediment pumping is especially evident for the 'waxing' condition GM1, which produces a gradual build-up of SSC during the wave group (Figure $10 \mathrm{a}, t / T_{\mathrm{gr}}=0.2$ to 0.85 ). Subsequently, a relatively long period with little wave action follows between $t / T_{\mathrm{gr}}=0.8$ and 0.1 into the next wave group $(|u|<0.2 \mathrm{~m} / \mathrm{s}$ for approximately 13 s). During this interval, most suspended sand settles out of suspension and the SSC decreases to nearly 0 upon the start of the next group (Figure 10a). Compared to the symmetric group GB1 (Figure 9a), the suspended load at the end of the wave group is higher for the waxing group GM1 (Figure 10a), hence suggesting that the latter is more effective in terms of sediment pumping. However, during two subsequent wave groups the suspended sediment settles out almost completely for GM1 whereas it remains partly in suspension for condition GB1. Consequently, as explored in more detail in Section 5.3, conditions GB1 and GM1 produce a similar time-averaged suspended sediment load. 
The 'waning' condition GM2 shows fluctuations at short-wave and wave group time scales, similar to the other wave group conditions (Figure $10 \mathrm{~b}$ ). The suspended load at the end of the group $\left(t / T_{\mathrm{gr}}=0.8\right.$ ) is lower for GM2 than for GM1. This suggests more effective sediment pumping for a 'waxing' group, where short-wave heights increase gradually and fall back rapidly, than for a 'waning' group. The same was concluded by O’Hara Murray et al. (2011) by studying SSC under random waves.

\subsection{SSC phase lags}

The phase behaviour of suspended sand is explored in more detail through the cross-correlation $R$ between water surface level $\eta(t)$ and suspended sand concentration $C(\zeta, t)$, calculated for each condition through:

$$
R(\zeta, \tau)=\frac{1}{N} \sum_{n=0}^{N-m-1}\left[\eta\left(t_{n}+\tau\right)-\bar{\eta}\right]\left[C\left(\zeta, t_{n}\right)-\bar{C}(\zeta)\right]
$$

Herein, $\Delta t=1 / 64 \mathrm{~s}$ is the temporal resolution of ABS measurements; $\tau=m \Delta t$ is a time displacement; $t_{\mathrm{n}}$ is the time index corresponding to the integer $n$; $N$ is the number of measurements in the time series. Cross-correlations are calculated for both the short waves and the wave groups. For the latter, the shortwave envelope of the water surface elevation, $\eta_{\mathrm{env}}$, was considered to be the most suitable parameter for analysing the phase behaviour of SSC. For consistency, the water surface elevation (rather than the velocity) was also used for the calculations of $R$ for the monochromatic waves. Equation (8) was evaluated using $\eta(t)$ measurements by the pressure transducer on the measurement frame, which were linearly interpolated to the same temporal resolution as the ABS data $\left(f_{\mathrm{s}}=64 \mathrm{~Hz}\right)$.

The cross-correlation results provide insights into the phase lag of SSC with respect to the water surface elevation. Positive $R(\zeta, \tau)$ indicates that SSC at height $\zeta$ and time lag $\tau$ is in phase with $\eta$; negative $R(\zeta$, $\tau$ ) indicates that $C(\zeta)$ at time lag $\tau$ is in anti-phase with $\eta$. Of particular interest is the time displacement $\tau_{\max }(\zeta)$ that corresponds to maximum $R(\zeta)$ : this time displacement, normalised by the wave period (i.e., $\left.\tau_{\max }(\zeta) / T\right)$, represents the phase lag of $C(\zeta)$ relative to the water surface elevation. An additional 
parameter of interest is the cross-correlation at zero phase lag, $R_{0}(\zeta)=R(\zeta, \tau=0)$. Note that $R_{0}(\zeta)>0$ indicates that $C(\zeta)$ is in phase with the water surface elevation, i.e., highest SSC under the wave crest, while $R_{0}(\zeta)<0$ indicates that SSC is maximum under the wave trough.

Figure 11 shows the cross-correlations for the monochromatic conditions M1 to M3 (top to bottom). The left-hand panels show $R(\zeta, \tau)$ as colour contours, with the white circles in each panel indicating the phase lag $\tau_{\max }(\zeta) / T$. To avoid false identification of phase lags, $\tau_{\max }$ values were truncated for $R<0.01$ $\mathrm{kg} / \mathrm{m}^{2}$. The right-hand panels show the $R_{0}$ profiles. Note that the phase behaviour of sand suspension close to the bed $\left(\zeta / \Delta_{\mathrm{r}}<3\right)$ is strongly affected by the phase of local bedforms (van der Werf et al., 2007; Hurther \& Thorne, 2011), hence leading to an increased uncertainty in the cross-correlation results at these elevations.

Figure 11a shows the vertical distribution of the phase lag for condition M1 (solid white circles). At the bed, $\tau_{\max } / T \approx 0.1$ indicates that sediment pickup lags the water surface elevation by about $0.1 T . \tau_{\max }$ increases gradually with distance from the bed as the entrained sediment spreads vertically. At $\zeta / \Delta_{\mathrm{r}} \approx 2$, the phase lag experiences a sudden, rapid increase from $\tau_{\max } / T \approx 0.2$ to 0.6 . This phase behaviour suggests that SSC is almost in phase with $\eta$ at $\zeta / \Delta_{\mathrm{r}}<2$ but SSC lags $\eta$ by more than half a wave cycle at $\zeta / \Delta_{\mathrm{r}}>2$. This may be explained by two vertically separated mixing regimes: a near-bed 'convective' mixing regime where coherent turbulent structures induce rapid vertical mixing (here found at $\zeta / \Delta_{\mathrm{r}}<2$ ) and a more gradual 'diffusive’ mixing regime at higher elevations (which dominates in the present experiment at $2<\zeta / \Delta_{\mathrm{r}}<5$ ) (see also Nielsen, 1992; Thorne et al., 2002).

The strong variation of SSC phase lags with height leads to a complex $R_{0}$ profile (Figure 11b), composed of a three-layer structure with alternating positive and negative constituents that respectively correspond to SSC that is on average in phase and in anti-phase with the water surface elevation. Very close to the bed $\left(\zeta / \Delta_{\mathrm{r}}<1\right)$, positive $R_{0}$ suggests that SSC is approximately in phase with the water surface elevation. Further from the bed, at $1<\zeta / \Delta_{\mathrm{r}}<4$, negative $R_{0}$ corresponds to the highest SSC occurring during the troughs of the waves due to a phase lag of about half a wave cycle. At even higher elevations $\left(\zeta / \Delta_{\mathrm{r}}>4\right)$, $R_{0}$ is positive again (although of small magnitude), corresponding to slightly higher SSC during the 
wave crest relative to the trough phase. The strong vertical variation of the phase behaviour of SSC with elevation, as reflected in $R_{0}(\zeta)$, shows that the local flux measured at a free-stream elevation of a few ripple heights above the bed can be a poor predictor for the magnitude and direction of the net depthintegrated flux. This reaffirms previous conclusions by e.g. Conley and Beach (2003) and emphasises the necessity of high-resolution measurements of SSC near the bed.

The cross-correlation and phase lag behaviour for conditions M2 and M3 are qualitatively similar to M1, yet quantitatively different (Figure 11c-f). Condition M2 yields phase lags of $\tau_{\max } / T \approx 0.35$ at $\zeta=$ $3 \Delta_{\mathrm{r}}$ (Figure 11c), which is consistent with the timing of the three intra-wave suspension events for this condition. The cross-correlation for condition M3 (Figure 11e) is dominated by the single main intrawave suspension event, which lags the water surface elevation by about 0.2 to $0.25 T$. Inter-comparison of $\tau_{\max }$ for M1 to M3 shows that the phase lag of SSC reduces with increasing wave height $H$. This indicates more rapid vertical mixing, which can be explained by stronger vertical advection by the orbital velocity and possibly by higher turbulence levels. The sudden vertical jump in $\tau_{\max }(\zeta) / T$ appears only for M1, possibly because of a lower vortex strength for conditions M2 and M3 due to the lower $d_{0} / \lambda_{\mathrm{r}}$ (as discussed in Section 5.1). Figure 11b,d,f shows that the differences in SSC phase lag are also reflected in the $R_{0}$ profiles. For M2, SSC is on average in anti-phase with $\eta$ (negative $R_{0}$ ) over a major fraction of the near-bed region. For M3, $R_{0} \approx 0$ at most elevations, because SSC is maximum during the crest-totrough zero crossing of the water surface elevation, i.e., when $\eta \approx 0$.

Next, the SSC phase lag is explored for the wave group conditions. This is done by examining the crosscorrelation $R$ between water level $\eta$ and SSC as before, and also by the cross-correlation $R_{\mathrm{hf}}$ between the high-pass filtered water level $\left(\eta_{\mathrm{hf}}\right)$ and concentration $\left(C_{\mathrm{hf}}\right)$, and the cross-correlation $R_{\text {env }}$ between the wave group envelope $\left(\eta_{\text {env }}\right)$ and low-pass filtered concentration $C_{\mathrm{lf}}$.

Figure 12 shows the cross-correlations $R, R_{\mathrm{hf}}$ and $R_{\text {env }}$ for condition GB1. The cross-correlation $R(\zeta, \tau)$ (Figure 12a) reveals a complex pattern with positive and negative values at short- and long-wave time scales. Around $\tau=0$, negative $R$ generally prevails due to SSC variations at the wave group time scale: 
the highest concentrations occur under the highest short waves, i.e., during the trough phase of the bound long wave. This also explains the negative $R_{0}$ near the bed at $\zeta / \Delta_{\mathrm{r}}<2$ (Figure 12b).

In order to better understand the distributions in Figure 12a-b, the cross-correlations at short- and longwave frequencies $R_{\mathrm{hf}}$ and $R_{\mathrm{env}}$ were explored. $R_{\mathrm{hf}}$ (Figure 12c) shows patterns that are similar to the monochromatic cases. Positive $R_{\mathrm{hf}}$ occurs near the bed around $\tau=0$ and spreads upward with increasing time displacement to $\tau / T_{\mathrm{m}} \approx 0.5$ at $1<\zeta / \Delta_{\mathrm{r}}<3$. A region of positive $R_{\mathrm{hf}}$ between $\zeta / \Delta_{\mathrm{r}}=1.5-10$ around $\tau / T_{\mathrm{m}}=0$ stands out. This region is not clearly connected to the red region at $\zeta / \Delta_{\mathrm{r}}<1$, which suggests that it is not induced by local sand pick-up but that it may instead be explained by suspended sand that is horizontally advected. Previous studies have indicated that cross-shore advection of suspended sand leads to an increase in SSC under the wave crest (Kranenburg et al., 2013), which would indeed be consistent with the positive $R_{\mathrm{hf}, 0}$ at these elevations (Figure 12d). This advection effect is more evident for condition GB1 than for the monochromatic conditions because of its relatively low wave skewness. The monochromatic waves, on the other hand, are characterised by a relatively high skewness, which induces a stronger intra-wave variation in SSC that dominates the calculated cross-correlation.

The cross-correlation at wave group frequency (Figure 12e-f) reveals positive $R_{\mathrm{env}}$ at $\tau / T_{\mathrm{gr}} \approx 0$, indicating highest SSC under the crest of the wave group envelope. Figure 12e (white markers) shows the phase lag at the wave group frequency. The phase lag at the bed is $\tau_{\max } \approx 0.1 T_{\mathrm{gr}} \approx T_{\mathrm{m}}$ and it increases with distance from the bed due to sediment pumping by the successive waves, yielding a value of $\tau \approx 0.2 T_{\mathrm{gr}}$ $\approx 2 T_{\mathrm{m}}$ at $\zeta \approx 8 \Delta_{\mathrm{r}}$. A similar magnitude and vertical distribution of the phase lag at wave group time scale was measured by O’Hara Murray et al. (2012) for random waves.

Figure 13 shows the cross-correlation for the other wave group conditions. For brevity, only the crosscorrelation at wave group frequency $R_{\text {env }}$ is shown. All conditions yield $R_{\text {env }}(\tau)$ and $\tau_{\max }(\zeta)$ distributions that are qualitatively similar to GB1. Condition GB2 results in a lower $\tau_{\max }$ at the bed than the other conditions, which indicates that suspended sediment pumping commences a bit earlier within the wave group. Moreover, this condition produces the highest steepness $\mathrm{d} \zeta / \mathrm{d} \tau_{\max }$ of all grouped waves, hence indicating the most rapid vertical spreading of suspended sediment. This may relate to the highly skewed 
and high short waves for GB2, which induce rapid vertical advective mixing of suspended sand. The phase lags for conditions GB1, GB3, GM1 and GM2 are similar for $\zeta / \Delta_{\mathrm{r}}<4$. At $\zeta / \Delta_{\mathrm{r}}>4$, conditions GB3 and GM2 yield higher phase lags than GB1 and GM1, hence indicating a more gradual vertical mixing of suspended sand.

\subsection{Time-averaged SSC and sediment diffusivity}

In equilibrium conditions, the time-averaged SSC profile $\bar{C}(\zeta)$ can be described as a balance between sand settling and upward vertical mixing. The latter can be described as a diffusive process, yielding (e.g. Nielsen, 1992):

$$
w_{\mathrm{s}} \bar{C}+\epsilon \frac{\mathrm{d} \bar{C}}{\mathrm{~d} \zeta}=0
$$

Here, $\epsilon$ is the local sediment diffusivity, which is linked to the turbulent viscosity but also includes the effects of vertical wave-related mixing (Nielsen, 1992). Another factor that controls $\bar{C}(\zeta)$ is the reference concentration $C_{\text {ref }}$ that defines the time-averaged sediment pickup from the bed.

In this section, the $\bar{C}(\zeta)$ distributions are analysed and related to $C_{\text {ref }}$ and $\epsilon(\zeta)$. Following van Rijn (2007), $C_{\text {ref }}$ is defined:

$$
C_{\text {ref }}=\bar{C}\left(\zeta_{\mathrm{a}}\right)
$$

where the reference level $\zeta_{\mathrm{a}}$ is defined (van Rijn, 2007) as:

$$
\zeta_{\mathrm{a}}=\max \left(\frac{\Delta_{\mathrm{r}}}{2}, 0.01 \mathrm{~m}\right)
$$

The reference concentration is directly calculated from the measurements using Equations (10) and (11). The sediment diffusivity distribution $\epsilon(\zeta)$ was obtained following the same approach as Thorne et al. (2009), that is, by solving Equation (9) numerically. The settling velocity $w_{\mathbf{s}}(\zeta)$ was estimated with the empirical formula of Soulsby (1997), using the power-law-distributed $D_{50}(\zeta)$ (Appendix A) as input. The vertical concentration gradient in Equation (9) was calculated over two vertical concentration measurement bins for $\zeta<0.20 \mathrm{~m}$ and over four vertical bins for $\zeta>0.20 \mathrm{~m}$. 
For reference, predictions of $\epsilon(\zeta)$ were calculated using the empirical model of van Rijn (1993):

$$
\begin{array}{cc}
\epsilon=\epsilon_{\text {bed }}=0.012 U_{0} \Delta_{\mathrm{r}} D_{50}[(s-1) g / v]^{1 / 3} & \text { for } \zeta \leq \zeta_{\mathrm{s}} \\
\epsilon=\epsilon_{\text {bed }}+\left(\epsilon_{\max }-\epsilon_{\text {bed }}\right)\left(\frac{\zeta-\zeta_{\mathrm{s}}}{0.5 h-\zeta_{\mathrm{s}}}\right) & \text { for } \zeta_{\mathrm{s}}<\zeta \leq 0.5 h \\
\epsilon=\epsilon_{\max }=0.035 H_{\mathrm{s}} h / T_{\mathrm{m}} \quad \text { for } \zeta>0.5 h
\end{array}
$$

583 Here, $U_{0}=\sqrt{2} \tilde{u}_{\text {rms }}$ is the representative orbital velocity amplitude; $s=2.65$ is the specific gravity of sand 584 in water; $g$ is the gravitational acceleration; $v=10^{-6} \mathrm{~m}^{2} / \mathrm{s}$ is the kinematic viscosity; $H_{\mathrm{s}}=4 \eta_{\text {rms }}$ is the significant wave height; $h$ is the local water depth. Equation (12) predicts vertically uniform $\epsilon(\zeta)$ over a near-bed mixing layer with thickness $\zeta_{\mathrm{s}}=3 \Delta_{\mathrm{r}}$ that is characterised by strong vertical mixing by bedshear-generated vortices. At higher elevations, $\epsilon(\zeta)$ increases linearly up to a maximum value $\epsilon_{\max }$ at $\zeta=$ $0.5 h$. The diffusivity in the upper half of the water column, $\epsilon_{\max }$, represents the vertical mixing by waves. Note that the bed-averaging method (Section 2.5) differs from the treatment by Van Rijn (1993, 2007), who uses a fixed, mean bed level. Therefore, the calculations were repeated using Van Rijn's bed level definition. This alternative approach does not lead to substantially different results, except near the bed (at $\zeta<2 \Delta_{\mathrm{r}}$ ) since Van Rijn’s definition requires truncation of the SSC profiles at a higher elevation.

Figure 14a,c,e shows the vertical distributions of $\bar{C}(\zeta)$ measured by ABS and TSS. Both instruments yield similar concentration profiles. The variability in $\zeta$ for the TSS measurements is considered to be the largest source of measurement uncertainty. Figure 14b,d,f shows the ABS measured (symbols) and predicted (dashed lines) $\epsilon(\zeta)$ distributions, normalised by $U_{0} \Delta_{\mathrm{r}}$ (following Thorne et al., 2009). Table 4 presents the measured reference concentrations and the depth-averaged diffusivities over the near-bed mixing layer. The measurement uncertainty of $C_{\text {ref }}$ was established by shifting the reference height $\zeta_{\mathrm{a}}$ by $5 \mathrm{~mm}$ (one vertical bin of the ABS) upward and downward. The uncertainty of $\epsilon$ in Table 4 was calculated as the standard deviation over the near-bed mixing layer.

The three monochromatic conditions produce $\bar{C}(\zeta)$ with similar shapes but different magnitudes (Figure 14a). Each profile increases exponentially (i.e. straight line on semi-log scale) near the bed (up to $\zeta / \Delta_{\mathrm{r}}$ $\approx 5$ for $\mathrm{M} 1$ and $\zeta / \Delta_{\mathrm{r}} \approx 3$ for M2 and M3) and is upward concave at higher elevations. Such concentration distributions are consistent with previous observations over rippled beds (Thorne et al., 2002; Vincent 
\& Hanes, 2002). As explained by e.g. Thorne et al. (2009), the near-bed exponentially increasing $\bar{C}(\zeta)$ corresponds to vertically constant $\epsilon(\zeta)$, while the upward concave $\bar{C}(\zeta)$ segment indicates a linearly increasing $\epsilon(\zeta)$. The measured $\epsilon(\zeta)$ distributions in Figure 14b confirm this two-layer structure, which is also conceptually consistent with van Rijn’s (1993) empirical model (Equation (12)).

Quantitatively, the three monochromatic conditions show clear differences. Firstly, the near-bed mixing layer thickness $\zeta_{\mathrm{s}}$, characterised by depth-uniform diffusivity, decreases with increasing wave height: $\zeta_{\mathrm{s}}$ $\approx 5 \Delta_{\mathrm{r}}$ for M1, $\zeta_{\mathrm{s}} \approx 3.5 \Delta_{\mathrm{r}}$ for M2, and $\zeta_{\mathrm{s}} \approx 2.5 \Delta_{\mathrm{r}}$ for M3 (Figure 14b). Secondly, the SSC in the near-bed layer decreases with increasing wave height (Figure 14a), which relates primarily to a decreasing reference concentration (Table 4). The decrease in suspended load and near-bed mixing layer thickness may physically relate to the reduction in $d_{0} / \lambda$ (Table 3), as discussed in Section 5.1 and in other studies (Malarkey \& Davies, 2002; O'Hara Murray et al., 2011).

Figure 14c-d shows $\bar{C}(\zeta)$ and $\epsilon(\zeta)$ for the bichromatic GB conditions. Both concentration and diffusivity profiles follow similar distributions as for the monochromatic waves. The corresponding $\epsilon(\zeta)$ profiles show a less abrupt transition from the near-bed mixing layer with depth-uniform $\epsilon$ to the section with vertically increasing $\epsilon$ than in Figure 14b, possibly due to contribution from the varying short waves within the group which smoothens the transition. In addition, the mixing layer thickness $\zeta_{s}$ is somewhat thicker than for the monochromatic waves, which possibly relates to a gradual build-up of bed-sheargenerated turbulence kinetic energy under the wave groups (Villard et al., 2000).

The three GB conditions produce $\bar{C}(\zeta)$ profiles that are nearly identical (Figure 14c). GB1 produces a higher suspended sand concentration far from the bed $\left(\zeta / \Delta_{\mathrm{r}}>8\right)$ but lower concentration close to the bed than GB2 and GB3. This may indicate more vertical mixing, i.e., more effective suspended sediment pumping, for condition GB1. However, the differences are small. The latter is also reflected in the reference concentrations and near-bed diffusivity for the GB conditions, which are very similar with differences within the measurement uncertainty (Table 4). 

revealed by the $20 \%$ higher $\epsilon /\left(U_{0} \Delta_{\mathrm{r}}\right)$ for GM1 than for GM2 (Figure 14f, Table 4). Conditions GM1 (waning) and GB1 (no saw-tooth modulation) produce almost identical $\bar{C}(\zeta)$ at $\zeta / \Delta_{\mathrm{r}}<10$, but GM1 produces higher concentrations further from the bed (Figure 14e). This difference is especially explained by the higher diffusivity at $\zeta / \Delta_{\mathrm{r}}>10$; the near-bed diffusivity is similar for GB1 and GM1 (Figure 14d,f, Table 4).

Comparing the wave group conditions with the monochromatic conditions shows that $\bar{C}(\zeta)$ is on average higher for the wave groups (Figure 14a,c,e). The wave group conditions also yield higher $\epsilon /\left(U_{0} \Delta_{\mathrm{r}}\right)$ than the monochromatic waves (Table 4). This could be attributed to sediment pumping by the groups. However, this result depends strongly on the vertical scaling of elevation with ripple height and the differences disappear when no scaling is applied.

Finally, the measured $\epsilon(\zeta)$ distributions are compared with the empirical predictions from Equation (12) (Figure 14b,d,f). For all conditions, the measurements and empirical predictions both follow a threelayer structure, which implies that Van Rijn's (1993) model is likely preferable over models that assume linearly increasing $\epsilon(\zeta)$ from the bed (e.g. Grant \& Madsen, 1979). Two main differences between the experimental observations and Van Rijn's model are observed. Firstly, the near-bed diffusivity over $\zeta=$ 0 to $3 \Delta_{\mathrm{r}}$ is overestimated for the monochromatic waves. For the present $D_{50}$, Equation (12) predicts $\epsilon_{\text {bed }} /\left(U_{0} \Delta_{\mathrm{r}}\right)=0.076$ for all conditions. A comparison with the measured data in Table 4 shows that $\epsilon_{\text {bed }} /\left(U_{0} \Delta_{\mathrm{r}}\right)$ is overestimated by 20 to $70 \%$ for the monochromatic waves. Note that van Rijn's model was based on measurements for ripples with higher steepness than those in the present experiment, which may explain some of the observed deviation between model and measurements. For the grouped wave conditions, which produce a higher near-bed diffusivity, $\epsilon_{\mathrm{bed}} /\left(U_{0} \Delta_{\mathrm{r}}\right)=0.076$ is in good agreement with the measurements (Table 4).

A second difference between modelled and measured diffusivity is the height of the near-bed mixing layer, $\zeta_{s}$, which appears to exceed $3 \Delta_{\mathrm{r}}$, especially for the grouped wave conditions. This may not relate entirely to wave group effects but also to a general uncertainty in $\zeta_{\mathrm{s}}$, since past measurements under regular waves also indicated $\zeta_{\mathrm{s}}>3 \Delta_{\mathrm{r}}$ (Thorne et al., 2009). Moreover, in a more recent version of his 
$=2 \delta_{\mathrm{w}}$, where $\delta_{\mathrm{w}}$ is an estimate of the wave bottom boundary layer thickness and is calculated as:

$$
\delta_{\mathrm{w}}=0.214 d_{0}^{0.75} \Delta_{\mathrm{r}}^{0.25}
$$

Figure 15 shows diffusivity profiles for all conditions, using different normalizations for the vertical axes. In Figure 15a the elevation is normalized by $\zeta_{\mathrm{s}}=3 \Delta_{\mathrm{r}}$. Significant variability in diffusivity distributions between conditions and a large discrepancy between measured and predicted diffusivity (solid line, for condition GB1) can be observed. If, instead, the elevation is normalized by $\zeta_{s}=2 \delta_{w}$, the variability between the conditions reduces significantly (igure 15b). Moreover, the measurements show significantly better agreement with the empirical prediction when $\zeta_{\mathrm{s}}=2 \delta_{\mathrm{w}}$ is assumed.

\subsection{Sand flux}

In this section the net (i.e. time-averaged) sand flux distributions $\overline{u(\zeta, t) C(\zeta, t)}$ are explored. Because no measurements of the velocity distribution $u(\zeta, t)$ were obtained, the flux distribution is estimated here as $\overline{u(t) C(\zeta, t)}$, where $u(t)$ is the free-stream velocity at $\zeta=0.16 \mathrm{~m}$. This means that the flux profiles do not consider the vertical variations in cross-shore velocity. This especially reduces the accuracy within the wave bottom boundary layer, where the phase and magnitude of the velocity are affected by the presence of the bed. Experiments in regular oscillatory flow showed that the phase and magnitude variations of velocity occur mainly within a thin layer of 1 ripple height above the bed (van der Werf et al., 2007). For that reason, the estimated flux is truncated for $\zeta<\Delta_{\mathrm{r}}$. Although the accordingly derived flux profiles may not be fully accurate, they are accepted for the primary objective in this section, which is to compare the various wave conditions in terms of net sand transport. The net flux $\overline{u_{0} C}$ was decomposed as

$$
\overline{u(t) C(\zeta, t)} \approx \bar{u}(t) \bar{C}(\zeta, t)+\overline{\left\langle\tilde{u}_{\mathrm{hf}}(t)\right\rangle\left\langle\widetilde{C}_{\mathrm{hf}}(\zeta, t)\right\rangle}+\overline{\left\langle\tilde{u}_{\mathrm{lf}}(t)\right\rangle\left\langle\widetilde{C}_{\mathrm{lf}}(\zeta, t)\right\rangle}
$$

where the three terms on the right-hand side define the net flux contributions by the current, the short waves, and the long (infra-gravity) wave. The turbulent diffusive flux $\overline{u^{\prime} C^{\prime}}$ could not be quantified on the basis of the present measurements and is not considered further. 
Figure 16a shows the vertical distributions of the net total flux for the monochromatic conditions. Flux magnitudes decrease rapidly with distance from the bed, with the strongest offshore-directed flux over $\zeta / \Delta_{\mathrm{r}}=1$ to 3 for all conditions. All conditions generate small net onshore fluxes over a short segment $\left(\zeta / \Delta_{\mathrm{r}} \approx 4\right.$ to 10 for M1; $\zeta / \Delta_{\mathrm{r}} \approx 4$ to 6 for M2 and M3); at higher elevations the net total flux returns to negative values. The different flux distributions for the three monochromatic conditions can be better understood by analysing the contributions of the current-related flux (Figure 16b) and wave-related flux (Figure 16c). The current-related flux is offshore for all conditions due do negative $\bar{u}$ (see Table 2 ). The magnitude and direction of the wave-related flux depend on the vertical and intra-wave temporal variation of SSC. Figure 16c shows alternating offshore- and onshore-directed constituents, which relate directly to the $R_{0}$ profile (Figure $11 \mathrm{~b}-\mathrm{d}-\mathrm{f}$ ). The wave-related fluxes at $\zeta / \Delta_{\mathrm{r}}<4$ are offshore because the SSC lags the velocity by approximately half a wave cycle at these elevations. Higher up, the waverelated flux is onshore because SSC is on average in phase with the water surface elevation. However, these onshore flux magnitudes are much smaller because the concentration decreases rapidly with distance from the bed.

The net fluxes for the wave group conditions are shown in Figure 17. Here, the total flux (Figure 17a) was decomposed into a current-related (Figure 17b), short-wave-related (Figure 17c) and long-waverelated (Figure 17d) contribution. Similar to the monochromatic conditions, the current-related component is offshore-directed due to $\bar{u}<0$. Strongest offshore current-related fluxes are produced by conditions GB2 and GB3, which relate to relatively high $|\bar{u}|$ for these conditions (Table 2). The shortwave-related component (Figure 17c) varies greatly between the various wave conditions. Condition GB1 produces an onshore short-wave flux over $\zeta / \Delta_{\mathrm{r}}=1-7$, which is attributed to the low velocity skewness of this condition (as explained in Section 5.2). The other conditions produce generally offshore-directed short-wave-related fluxes, with greatest magnitudes for the partially-modulated condition GB3.

Figure 17d shows the net flux by the long wave, driven by the phase coupling of SSC at the wave group time scale. The long-wave flux is offshore-directed for all conditions, which agrees with previous sand flux observations under non-breaking wave groups in field (e.g. Shi and Larsen, 1984) and laboratory 
(e.g. Sato, 1992) conditions. The differences in flux magnitudes between the conditions are explained by the timing of the 'pumped' suspended load relative to the phase behaviour of the long-wave velocity $\left\langle\tilde{u}_{0, \text { If }}\right\rangle$. For conditions GB1 and GM1, this timing leads to the greatest magnitudes of the net long-wave flux.

\subsection{Net suspended sand transport rates}

The conditions are further compared in terms of net sand transport rates. The net depth-integrated suspended transport rate $q_{\mathrm{s}}$ is calculated here as

$$
q_{\mathrm{S}}=\int_{\Delta_{\mathrm{r}}}^{0.50} \overline{\left\langle u_{0}(t)\right\rangle\langle C(\zeta, t)\rangle} \mathrm{d} \zeta
$$

The upper bound of $\zeta=0.50 \mathrm{~m}$ is chosen here for pragmatic reasons as the highest elevation at which the SSC measurements are considered unaffected by the mounting arrangement for the ABS transducers at $\zeta=0.82 \mathrm{~m}$. Note that the present experimental set-up does not allow quantification of sand transport over the whole water column, and the accordingly obtained $q_{\mathrm{s}}$ neglects the transport contributions at $\zeta<$ $\Delta_{\mathrm{r}}$ and $\zeta>0.50 \mathrm{~m}$. For the objective of analysing the differences in net suspended transport rates between the various wave conditions, this is considered a reasonable approach.

Figure 18 shows the net suspended sand transport rates, decomposed into current-, short-wave-, and long-wave-related contributions. It directly follows that all transport components are of similar order of magnitude and are therefore significant to the total suspended transport. All wave group conditions (GB and GM) generate offshore-directed total $q_{\mathrm{s}}$, yet the magnitudes differ substantially. The offshore transport is relatively low for GB1 and relatively high for GB3, while the other conditions (GB2, GM1, and GM2) yield approximately similar total $q_{\mathrm{s}}$. These differences are primarily caused by variations in the short-wave-related transport, which is onshore-directed for GB1 and is offshore-directed for GB3. The GB and GM conditions also differ in terms of current-related and long-wave-related transport rates, but these differences are small compared to those observed for the short-wave-related transport. The 
three monochromatic conditions produce total $q_{\mathrm{s}}$ that is of similar magnitude to the wave group conditions. Magnitudes of $q_{\mathrm{s}}$ increase from M1 to M2 due to an increase in suspended load with increasing wave height, but decrease from M2 to M3 due to the reduced slopes of bedforms for the latter condition.

The equivalence between the monochromatic and wave group conditions in terms of transport rates is discussed in the next section.

\section{Discussion}

The experimental program was designed to study the differences in suspended sand concentration and transport rates for various 'equivalent' wave groups and monochromatic conditions. The five wave groups produced similar $\eta_{\text {rms }}, \tilde{u}_{\text {rms }}$, and $d_{0}$ over the test section, yet differed in terms of velocity skewness $S k(\tilde{u})$ due to variations in wave shape transformation during the shoaling process. These differences complicate the interpretation of the measurements in the present experiment, especially the phase behaviour of SSC at intra-wave time scale and the net short-wave-related flux, which are both strongly affected by the degree of velocity skewness (see e.g. van der Werf et al., 2007; Amoudry et al., 2016). As a result, the differences in net short-wave-related and net total transport rates between the various GB conditions (Section 5.5) cannot be attributed solely to wave group shape (i.e. wave group duration and degree of modulation), but also to differences in $S k(\tilde{u})$. Consequently, the effects of wave group duration and degree of modulation on intra-wave SSC and on net short-wave-related suspended sand transport cannot be fully analysed on the basis of the present data, leaving this topic partly open for future experimental and/or numerical research.

The 'equivalent' monochromatic conditions also deviate from the wave group conditions in some respects, producing flows with higher $\tilde{u}_{\mathrm{rms}}, d_{0}$ and $S k(\tilde{u})$ than their wave group equivalents. An additional complicating factor is that the monochromatic condition M2 and M3 produced significantly higher and longer bedforms than the wave groups. 
While taking these differences into consideration, the bichromatic wave conditions are compared to their monochromatic equivalents in terms of time-averaged SSC. The wave group conditions lead to generally higher time-averaged SSC than the monochromatic waves. This is explained by a similar reference concentration $C_{\text {ref }}$ for all wave conditions, but a higher non-dimensional near-bed sediment diffusivity $\epsilon_{\text {bed }} /\left(U_{0} \Delta_{\mathrm{r}}\right)$ for the grouped wave conditions. The latter may reflect a sediment pumping effect over the wave groups that is absent for the monochromatic waves. Such pumping effects could be accounted for in numerical models by means of an empirical factor $\beta_{\mathrm{gr}}$ in the formulation for the near-bed diffusivity, e.g.:

$$
\epsilon_{\text {bed, gr }}=\beta_{\text {gr }} \epsilon_{\text {bed }}
$$

Here, $\epsilon_{\text {bed,gr }}$ is the near-bed diffusivity for wave groups. A value for the wave group correction $\beta_{\text {gr }}$ in the range of 1.3 to 1.9 is proposed based on the present observations.

The wave group and monochromatic conditions can be further compared in terms of net suspended sand flux and transport rates. The various wave groups (GB and GM) result in notably different net suspended sand transport rates, with $q_{\mathrm{s}}$ varying by up to a factor 3 (see Section 6). This is especially attributed to variations in the short-wave suspended transport component, which varies significantly for the different wave group conditions due to variability in the short-wave skewness. All three equivalent monochromatic conditions produce $q_{\mathrm{s}}$ that is within the $q_{\mathrm{s}}$ variability of the wave group conditions. This happens even though the processes driving net suspended sand transport are fundamentally different for the wave groups versus the monochromatic waves; for instance, there is no transport by the long wave for the monochromatic conditions.

In summary, the present data suggest that an equivalent wave concept is applicable for suspended sand distributions and suspended sand transport rates, although a modification to near-bed diffusivity formulations is proposed to account for sediment pumping effects. The time-averaged suspended sand concentrations are best represented by a monochromatic wave with $\eta_{\text {rms }}$ equal to the wave groups (condition M1). 
Based on these results, further development of numerical modelling concepts for irregular waves would particularly benefit from the development of parameterizations for wave group shape, duration, and short-wave skewness effects on the time-averaged diffusivity. The results further stress the importance of accurate model formulations for the short-wave-driven suspended sand transport rate, which shows, from all suspended transport components, the strongest variability between wave conditions. The presented data are made publicly available to facilitate model validation and development. In addition, field measurements may verify the relationships between sand transport and wave group characteristics (duration, degree of modulation, and short wave sequencing) observed in the present experiments.

\section{Conclusions}

The present study focused on sand transport dynamics by five wave groups, and three monochromatic wave conditions that had the same mean period and 'equivalent' root-mean-square water surface elevation or maximum wave height as the wave groups. Experiments were conducted in a large-scale wave flume, involving non-breaking waves propagating over a horizontal medium-sand bed. All wave conditions produced sand ripples in a post-vortex regime. Measurements were obtained of the water surface elevation, free-stream velocity, suspended sand concentration (SSC) profiles, and bedform dimensions and migration rates.

The five grouped wave conditions produce similar time-averaged SSC profiles, near-bed time-averaged sediment diffusivity, and reference concentration. On the other hand, the groups differ in terms of time variation of SSC. The symmetric group and the backward leaning 'waxing' group are the most effective in terms of sediment pumping as they produce the highest SSC at the end of the group. However, for these conditions, the suspended sand settles out almost completely between two consecutive groups, which explains why the differences in time-averaged SSC profiles between all groups are small.

The SSC phase lag at short-wave time scale, for both the grouped and monochromatic wave conditions, depends strongly on velocity skewness and bedform dimensions. The lag varies importantly with distance from the bed, reflecting the vertical spreading of suspended sand. At wave group time scale, 
SSC lags the wave envelope by one wave cycle at the bed and the phase lag increases approximately

808 linearly upward. The vertical mixing of suspended sand is the most rapid for the shortest wave group, 809 while the other groups generate similar SSC phase lags.

810

811

812

813

814

815

816

All wave group conditions produce net offshore-directed suspended sand transport, but magnitudes vary by a factor three. All transport components (current, short wave, long wave) contribute significantly to the total net suspended sand transport. Differences in the total net suspended sand transport between the five grouped wave conditions are primarily caused by variations in the short-wave-related sand transport rate.

The wave group conditions lead to consistently higher non-dimensional near-bed sediment diffusivity than the 'equivalent' monochromatic waves. The monochromatic wave with root-mean-square water surface elevation similar to that of the wave group conditions produces time-averaged SSC and diffusivity distributions that match closest to the observations for the grouped waves. The net suspended sand transport rate for this monochromatic wave is within the variability of the transport rates for the wave groups. This suggests that, for the present conditions, the equivalent wave concept seems applicable for time-averaged SSC distributions and net suspended transport. An empirical correction factor to the near-bed diffusivity is suggested to account for sediment pumping effects and to further improve numerical predictions of SSC and suspended sand transport under wave groups.

\section{Appendix A: grain sorting}

The vertical distributions of the median grain diameter $D_{50}$ of the suspended sand samples is shown in Figure A1. The samples do not show a clear differentiation between the various wave conditions. The $D_{50}$ data suggest a power-law distribution, i.e.:

$$
D_{50}(\zeta)=D_{50, \text { bed }}\left(\frac{\zeta}{\zeta}\right)^{-n}
$$


where $\zeta_{\mathrm{r}}=0.01 \mathrm{~m}$ is a reference elevation and the exponent $n$ is a vertical decay parameter. The latter was calibrated using the $D_{50}$ measurements for all conditions, yielding $n=0.23$ as best fit. The fitted power-law distribution is included in Figure A1.

\section{Acknowledgments}

We thank the technical staff of the UPC CIEMLAB and Sjoerd van Til for their contributions to the experiments, and the editor and an anonymous reviewer for their useful feedback on the manuscript.

The experimental work was supported by the European Community's Seventh Framework Programme through the grant to the budget of the Integrating Activity HYDRALAB IV within the Transnational Access Activities, Contract no. 261520, with additional funding from the Dutch Technology Foundation STW (project 12058) and the UK’s Engineering and Physical Sciences Research Council (EPSRC, grant number EP/J00507X/1) through the SINBAD project. JvdZ was funded through the European Community’s H2020 Programme HYDRALAB+ (Contract no. 654110). The presented data are stored in the 4TU online data repository (https://doi.org/10.4121/uuid:30496cc3-9803-4c18-8a6f85513bb29c3d).

\section{References}

Amoudry, L. O., Souza, A. J., Thorne, P. D., \& Liu, P. L. F. (2016). Parameterization of intrawave ripple-averaged sediment pickup above steep ripples. Journal of Geophysical Research: Oceans, 121(1), 658-673. doi:10.1002/2015jc011185

Bagnold, R. A. (1946). Motion of Waves in Shallow Water. Interaction between Waves and Sand Bottoms. Proceedings of the Royal Society A: Mathematical, Physical and Engineering Sciences, 187(1008), 1-18. doi:10.1098/rspa.1946.0062

Battjes, J. A., Bakkenes, H. J., Janssen, T. T., \& van Dongeren, A. R. (2004). Shoaling of subharmonic gravity waves. Journal of Geophysical Research, 109(C2). doi:10.1029/2003jc001863

Beach, R. A., \& Sternberg, R. W. (1988). Suspended sediment transport in the surf zone: Response to cross-shore infragravity motion. Marine Geology, 80(1-2), 61-79. doi:10.1016/00253227(88)90072-2

Bell, P. S., \& Thorne, P. D. (1997a). Measurement of sea bed evolution in an estuarine environment using a high resolution acoustic sand ripple profiling system. MTS/IEEE Conference Proceedings Oceans '97, Halifax, Canada.

Bell, P. S., \& Thorne, P. D. (1997b). Application of a high resolution acoustic scanning system for imaging sea bed microtopography. Seventh International Conference on Electronic Engineering in Oceanography. 
Bell, P. S., Thorne, P. D., \& Williams, J. J. (1998). Acoustic measurements of sand ripple profile evolution under controlled wave conditions. Proceedings of the fourth European Conference on Underwater Acoustics, Rome, Italy.

Blott, S. J., \& Pye, K. (2001). GRADISTAT: a grain size distribution and statistics package for the analysis of unconsolidated sediments. Earth Surface Processes and Landforms, 26(11), 12371248. doi:10.1002/esp.261

Bosman, J. J., van der Velden, E. T. J. M., \& Hulsbergen, C. H. (1987). Sediment concentration measurement by transverse suction. Coastal Engineering, 11(4), 353-370. doi:10.1016/03783839(87)90033-0

Clifton, H. E., \& Dingler, J. R. (1984). Wave-formed structures and paleoenvironmental reconstruction. Marine Geology, 60(1-4), 165-198. doi:10.1016/0025-3227(84)90149-х

Conley, D. C., \& Beach, R. A. (2003). Cross-shore sediment transport partitioning in the nearshore during a storm event. Journal of Geophysical Research, 108(C3). doi:10.1029/2001jc001230

Davies, A. G., \& Villaret, C. (1997). Oscillatory Flow over Rippled Beds: Boundary Layer Structure and Wave-Induced Eulerian Drift. In J. N. Hunt (Ed.), Gravity Waves in Water of Finite Depth (Vol. 10, pp. 215-254): Computational Mechanics Publications.

Davies, A. G., \& Villaret, C. (2002). Prediction of sand transport rates by waves and currents in the coastal zone. Continental Shelf Research, 22(18-19), 2725-2737. doi:10.1016/s02784343(02)00123-1

Davies, A. G., \& Thorne, P. D. (2016). On the suspension of graded sediment by waves above ripples: Inferences of convective and diffusive processes. Continental Shelf Research, 112, 46-67. doi:10.1016/j.csr.2015.10.006

de Bakker, A. T. M., Brinkkemper, J. A., van der Steen, F., Tissier, M. F. S., \& Ruessink, B. G. (2016). Cross-shore sand transport by infragravity waves as a function of beach steepness. Journal of Geophysical Research: Earth Surface, 121(10), 1786-1799. doi:10.1002/2016jf003878

Deigaard, R., Jakobsen, J. B., \& Fredsøe, J. (1999). Net sediment transport under wave groups and bound long waves. Journal of Geophysical Research, 104(C6), 13559. doi:10.1029/1999jc900072

Dohmen-Janssen, C. M., \& Hanes, D. M. (2005). Sheet flow and suspended sediment due to wave groups in a large wave flume. Continental Shelf Research, 25(3), 333-347. doi:10.1016/j.csr.2004.10.009

Finn, J. R., Li, M., \& Apte, S. V. (2016). Particle based modelling and simulation of natural sand dynamics in the wave bottom boundary layer. Journal of Fluid Mechanics, 796, 340-385. doi:10.1017/jfm.2016.246

Fredsøe, J., Andersen, K. H., \& Mutlu Sumer, B. (1999). Wave plus current over a ripple-covered bed. Coastal Engineering, 38(4), 177-221. doi:10.1016/s0378-3839(99)00047-2

Grant, W. D., \& Madsen, O. S. (1979). Combined wave and current interaction with a rough bottom. Journal of Geophysical Research: Oceans, 84(C4), 1797-1808. doi:10.1029/JC084iC04p01797

Guza, R. T., \& Thornton, E. B. (1980). Local and Shoaled Comparisons of Sea-Surface Elevations, Pressures, and Velocities. Journal of Geophysical Research-Oceans, 85(Nc3), 1524-1530. doi:DOI 10.1029/JC085iC03p01524

Hanes, D. M., \& Huntley, D. A. (1986). Continuous Measurements of Suspended Sand Concentration in a Wave Dominated Nearshore Environment. Continental Shelf Research, 6(4), 585-596.

Hanes, D. M. (1991). Suspension of Sand Due to Wave Groups. Journal of Geophysical ResearchOceans, 96(C5), 8911-8915. doi:Doi 10.1029/91jc00571

Hay, A. E., \& Bowen, A. J. (1994). Coherence scales of wave-induced suspended sand concentration fluctuations. Journal of Geophysical Research, 99(C6), 12749. doi:10.1029/94jc00290

Hoekstra, P., Bell, P., van Santen, P., Roode, N., Levoy, F., \& Whitehouse, R. (2004). Bedform migration and bedload transport on an intertidal shoal. Continental Shelf Research, 24(11), 1249-1269. doi:10.1016/j.csr.2004.03.006

Holmedal, L. E., Myrhaug, D., \& Eidsvik, K. J. (2004). Sediment suspension under sheet flow conditions beneath random waves plus current. Continental Shelf Research, 24(17), 2065-2091. doi:10.1016/j.csr.2004.06.021 
Hurther, D., \& Thorne, P. D. (2011). Suspension and near-bed load sediment transport processes above a migrating, sand-rippled bed under shoaling waves. Journal of Geophysical Research, 116(C7). doi:10.1029/2010jc006774

Jaffe, B. E., Sternberg, R. W., \& Sallenger, A. H. (1984). The role of suspended sediment in shorenormal beach profile changes. 19th International Conference on Coastal Engineering.

Janssen, T. T., Battjes, J. A., \& van Dongeren, A. R. (2003). Long waves induced by short-wave groups over a sloping bottom. Journal of Geophysical Research-Oceans, 108(C8), 1-14. doi:10.1029/2002jc001515

Kranenburg, W. M., Ribberink, J. S., Schretlen, J. J. L. M., \& Uittenbogaard, R. E. (2013). Sand transport beneath waves: The role of progressive wave streaming and other free surface effects. Journal of Geophysical Research: Earth Surface, 118(1), 122-139. doi:10.1029/2012jf002427

Longuet-Higgins, M. S., \& Stewart, R. W. (1962). Radiation stress and mass transport in gravity waves, with application to 'surf beats'. Journal of Fluid Mechanics, 13(04), 481. doi:10.1017/s0022112062000877

Malarkey, J., \& Davies, A. G. (2002). Discrete vortex modelling of oscillatory flow over ripples. Applied Ocean Research, 24(3), 127-145. doi:10.1016/s0141-1187(02)00035-4

Malarkey, J., \& Davies, A. G. (2012). Free-stream velocity descriptions under waves with skewness and asymmetry. Coastal Engineering, 68, 78-95. doi:10.1016/j.coastaleng.2012.04.009

Nakato, T., Locher, F. A., Glover, J. R., \& F., K. J. (1977). Wave Entrainment of Sediment from Ripple Beds. Journal of the waterway Port Coastal and Ocean Division, 103(1), 83-99.

Nielsen, P. (1984). Field-Measurements of Time-Averaged Suspended Sediment Concentrations under Waves. Coastal Engineering, 8(1), 51-72. doi:10.1016/0378-3839(84)90022-X

Nielsen, P. (1992). Coastal Bottom Boundary Layers and Sediment Transport (Vol. 4). Singapore: World Scientific.

O'Donoghue, T., Doucette, J. S., van der Werf, J. J., \& Ribberink, J. S. (2006). The dimensions of sand ripples in full-scale oscillatory flows. Coastal Engineering, 53(12), 997-1012. doi:10.1016/j.coastaleng.2006.06.008

O'Hara Murray, R. B., Thorne, P. D., \& Hodgson, D. M. (2011). Intrawave observations of sediment entrainment processes above sand ripples under irregular waves. Journal of Geophysical Research, 116(C1). doi:10.1029/2010jc006216

O'Hara Murray, R. B., Hodgson, D. M., \& Thorne, P. D. (2012). Wave groups and sediment resuspension processes over evolving sandy bedforms. Continental Shelf Research, 46, 16-30. doi:DOI 10.1016/j.csr.2012.02.011

Osborne, P. D., \& Greenwood, B. (1992). Frequency-Dependent Cross-Shore Suspended Sediment Transport .1. A Non-Barred Shoreface. Marine Geology, 106(1-2), 1-24. Osborne, P. D., \& Greenwood, B. (1993). Sediment suspension under waves and currents: time scales and vertical structure. Sedimentology, 40(4), 599-622. doi:10.1111/j.1365-3091.1993.tb01352.x

Osborne, P. D., \& Vincent, C. E. (1996). Vertical and horizontal structure in suspended sand concentrations and wave-induced fluxes over bedforms. Marine Geology, 131(3-4), 195-208. doi:10.1016/0025-3227(95)00002-x

Padilla, E. M., \& Alsina, J. M. (2017). Transfer and dissipation of energy during wave group propagation on a gentle beach slope. Journal of Geophysical Research: Oceans, 122(8), 6773-6794. doi:10.1002/2017jc012703

Ribberink, J. S., \& Al-Salem, A. A. (1994). Sediment transport in oscillatory boundary layers in cases of rippled beds and sheet flow. Journal of Geophysical Research, 99(C6), 12707 - 12727. doi:10.1029/94jc00380

Ribberink, J. S., \& Al-Salem, A. A. (1995). Sheet Flow and Suspension of Sand in Oscillatory Boundary-Layers. Coastal Engineering, 25(3-4), 205-225. doi:10.1016/0378-3839(95)00003-T

Ribberink, J. S., van der A, D. A., van der Zanden, J., O'Donoghue, T., Hurther, D., Cáceres, I., \& Thorne, P. D. (2014). SandT-Pro: Sediment transport measurements under irregular and breaking waves. Proceedings of the 34th International Conference on Coastal Engineering, Seoul, Korea, Seoul, Korea. 10.9753/icce.v34.sediment.1

Ruessink, B. G., Houwman, K. T., \& Hoekstra, P. (1998). The systematic contribution of transporting mechanisms to the cross-shore sediment transport in water depths of 3 to $9 \mathrm{~m}$. Marine Geology, 
152(4), 295-324. doi:10.1016/S0025-3227(98)00133-9Sato, S. (1987). Oscillatory boundary layer flow and sand movement over ripples. PhD thesis, University of Tokyo, Japan.

Sato, S., Mitani, K., \& Watanabe, A. (1988). Geometry of Sand Ripples and Net Sand Transport Rate Due to Regular and Irregular Oscillatory Flows. Coastal Engineering in Japan, 30(2), 89-98. doi:10.1080/05785634.1988.11924477

Sato, S. (1992). Sand Transport under Grouping Waves. Proc. 23rd Int. Conf. on Coast. Eng., Venice, Italy.

Shi, N. C., \& Larsen, L. H. (1984). Reverse sediment transport induced by amplitude-modulated waves. Marine Geology, 54(3-4), 181-200. doi:10.1016/0025-3227(84)90037-9

Sleath, J. F. A. (1982). The Suspension Of Sand By Waves. Journal of Hydraulic Research, 20(5), 439452. doi:10.1080/00221688209499472

Soulsby, R. L. (1997). Dynamics of Marine Sands. London, U.K.: Thomas Telford.

Thorne, P. D., Williams, J. J., \& Davies, A. G. (2002). Suspended sediments under waves measured in a large-scale flume facility. Journal of Geophysical Research-Oceans, 107(C8). doi:10.1029/2001jc000988

Thorne, P. D., Davies, A. G., \& Williams, J. J. (2003). Measurements of near-bed intra-wave sediment entrainment above vortex ripples. Geophysical Research Letters, 30(20), n/a-n/a. doi:10.1029/2003gl018427

Thorne, P. D., Davies, A. G., \& Bell, P. S. (2009). Observations and analysis of sediment diffusivity profiles over sandy rippled beds under waves. Journal of Geophysical Research, 114(C02023), 1-16. doi:10.1029/2008JC004944

Thorne, P. D., \& Hurther, D. (2014). An overview on the use of backscattered sound for measuring suspended particle size and concentration profiles in non-cohesive inorganic sediment transport studies. Continental Shelf Research, 73, 97-118. doi:DOI 10.1016/j.csr.2013.10.017

Traykovski, P., Hay, A. E., Irish, J. D., \& Lynch, J. F. (1999). Geometry, migration, and evolution of wave orbital ripples at LEO-15. Journal of Geophysical Research: Oceans, 104(C1), 15051524. doi:10.1029/1998jc900026

van den Berg, J. H. (1987). Bedform migration and bed-load transport in some rivers and tidal environments. Sedimentology, 34(4), 681-698. doi:10.1111/j.1365-3091.1987.tb00794.x

van der Werf, J. J., Ribberink, J. S., O'Donoghue, T., \& Doucette, J. S. (2006). Modelling and measurement of sand transport processes over full-scale ripples in oscillatory flow. Coastal Engineering, 53(8), 657-673. doi:10.1016/j.coastaleng.2006.02.002

van der Werf, J. J., Doucette, J. S., O'Donoghue, T., \& Ribberink, J. S. (2007). Detailed measurements of velocities and suspended sand concentrations over full-scale ripples in regular oscillatory flow. Journal of Geophysical Research, 112(F2). doi:10.1029/2006jf000614

van der Zanden, J., van der A, D. A., Hurther, D., Cáceres, I., O’Donoghue, T., \& Ribberink, J. S. (2017). Suspended sediment transport around a large-scale laboratory breaker bar. Coastal Engineering, 125, 51-69. doi:10.1016/j.coastaleng.2017.03.007

van Rijn, L. C. (1993). Principles of Sediment Transport in Rivers, Estuaries and Coastal Seas. the Netherlands: Aqua Publications, the Netherlands.

van Rijn, L. C. (2007). Unified view of sediment transport by currents and waves. II: Suspended Transport. Journal of Hydraulic Engineering, 133(6), 668-689. doi:10.1061/(asce)07339429(2007)133:6(668)

van Rijn, L. C., Ribberink, J. S., van der Werf, J. J., \& Walstra, D. J. R. (2013). Coastal sediment dynamics: recent advances and future research needs. Journal of Hydraulic Research, 51(5), 475-493. doi:10.1080/00221686.2013.849297

Villard, P. V., Osborne, P. D., \& Vincent, C. E. (2000). Influence of wave groups on SSC patterns over vortex ripples. Continental Shelf Research, 20(17), 2391-2410. doi:10.1016/s02784343(99)00068-0

Vincent, C. E., Marsh, S. W., Webb, M. P., \& Osborne, P. D. (1999). Spatial and temporal structures of suspension and transport over megaripples on the shore face. Journal of Geophysical Research: Oceans, 104(C5), 11215-11224. doi:10.1029/1999jc900020

Vincent, C. E., \& Hanes, D. M. (2002). The accumulation and decay of near-bed suspended sand concentration due to waves and wave groups. Continental Shelf Research, 22(14), 1987-2000. doi:10.1016/s0278-4343(02)00051-1 
Wiberg, P. L., \& Harris, C. K. (1994). Ripple geometry in wave-dominated environments. Journal of Geophysical Research, 99(C1), 775. doi:10.1029/93jc02726

Williams, J. J., Rose, C. P., \& Thorne, P. D. (2002). Role of wave groups in resuspension of sandy sediments. Marine Geology, 183(1-4), 17-29. doi:10.1016/S0025-3227(01)00251-1

Williams, J. J., \& Bell, P. S. (2006). Laboratory investigation of bedform dynamics and resuspension of 1032 
1037 Table 1. Overview of wave conditions. $f_{1}$ and $f_{2}$ are the frequencies of the bichromatic wave components; $1038 T_{\mathrm{m}}$ is the mean short-wave period; $T_{\mathrm{gr}}$ is the wave group period; $N$ is the number of short waves in the 1039 group; $H_{1}$ and $H_{2}$ are the measured wave heights of the two bichromatic wave components; $\eta_{\text {rms }}$ and $1040 H_{\max }$ are the measured root-mean-square water surface elevation and maximum wave height. All water 1041 surface level parameters are measured near the wave paddle $(x=6.3 \mathrm{~m})$. Also indicated is during which 1042 runs continuous SRP, ABS and TSS measurements were made.

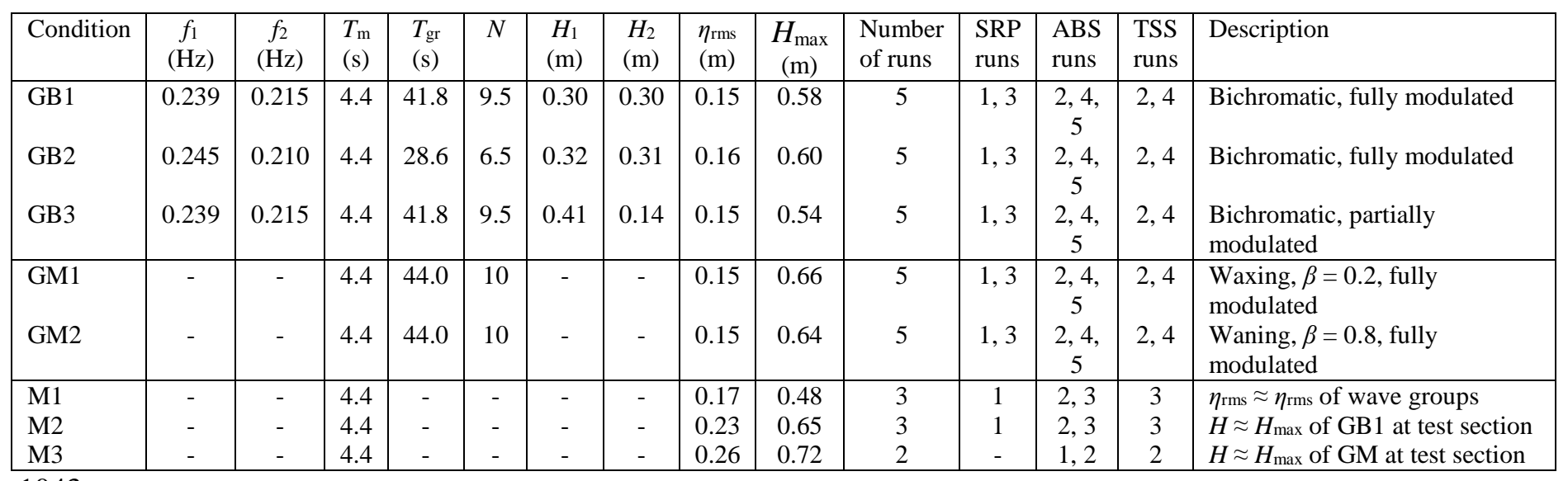

1043

1044

1045

1046 
Table 2. Measured hydrodynamic parameters at the test section. The water surface level measurements were obtained by an RWG, the velocity measurements were recorded by an ADV at $\zeta \approx 0.16 \mathrm{~m}$.

\begin{tabular}{|c|c|c|c|c|c|c|c|c|c|c|c|}
\hline Condition & $\begin{array}{c}\langle\eta\rangle_{\mathrm{rms}} \\
(\mathrm{m})\end{array}$ & $\begin{array}{c}\langle\eta\rangle_{\max } \\
(\mathrm{m})\end{array}$ & $\begin{array}{c}\langle\eta\rangle_{\min } \\
(\mathrm{m})\end{array}$ & $\begin{array}{c}H_{\max } \\
(\mathrm{m})\end{array}$ & $\begin{array}{c}\bar{u} \\
(\mathrm{~m} / \mathrm{s})\end{array}$ & $\begin{array}{c}\langle u\rangle_{\max } \\
(\mathrm{m} / \mathrm{s})\end{array}$ & $\begin{array}{c}\langle u\rangle_{\min } \\
(\mathrm{m} / \mathrm{s})\end{array}$ & $\begin{array}{c}\tilde{u}_{\mathrm{rms}} \\
(\mathrm{m} / \mathrm{s})\end{array}$ & $\begin{array}{c}S k(\langle\tilde{u}\rangle) \\
d_{0} \\
(\mathrm{~m})\end{array}$ & $\begin{array}{c}d_{0, \max } \\
(\mathrm{m})\end{array}$ \\
\hline GB1 & 0.17 & 0.61 & -0.22 & 0.83 & -0.016 & 0.67 & -0.59 & 0.31 & 0.27 & 0.56 & 1.08 \\
GB2 & 0.18 & 0.66 & -0.22 & 0.88 & -0.021 & 0.84 & -0.49 & 0.32 & 0.79 & 0.57 & 0.91 \\
GB3 & 0.18 & 0.52 & -0.18 & 0.70 & -0.023 & 0.69 & -0.52 & 0.33 & 0.54 & 0.61 & 0.93 \\
\hline GM1 & 0.16 & 0.68 & -0.25 & 0.93 & -0.012 & 0.82 & -0.56 & 0.30 & 0.44 & 0.53 & 1.14 \\
GM2 & 0.16 & 0.68 & -0.24 & 0.92 & -0.018 & 0.75 & -0.58 & 0.31 & 0.38 & 0.53 & 1.22 \\
\hline M1 & 0.18 & 0.41 & -0.16 & 0.58 & -0.012 & 0.69 & -0.37 & 0.35 & 0.77 & 0.67 & 0.67 \\
M2 & 0.23 & 0.58 & -0.21 & 0.79 & -0.060 & 0.83 & -0.47 & 0.42 & 0.94 & 0.78 & 0.78 \\
M3 & 0.27 & 0.73 & -0.24 & 0.97 & -0.071 & 0.93 & -0.54 & 0.45 & 0.99 & 0.83 & 0.83 \\
\hline
\end{tabular}

1049

1050 
1051 Table 3. Measured mean ripple height $\Delta_{\mathrm{r}}$, ripple length $\lambda_{\mathrm{r}}$, steepness $\Delta_{\mathrm{r}} / \lambda_{\mathrm{r}}$, and migration speed $c_{\mathrm{r}}$. 1052 Brackets denote standard deviation.

\begin{tabular}{|c|c|c|c|c|c|c|c|l|}
\hline Condition & $\begin{array}{c}\Delta_{\mathrm{r}} \\
(\mathrm{m})\end{array}$ & $\begin{array}{c}\lambda_{\mathrm{r}} \\
(\mathrm{m})\end{array}$ & $\Delta_{\mathrm{r}} / \lambda_{\mathrm{r}}$ & $\begin{array}{c}c_{\mathrm{r}} \\
(\mathrm{mm} / \mathrm{s})\end{array}$ & $d_{0} / \Delta_{\mathrm{r}}$ & $d_{0} / \lambda_{\mathrm{r}}$ & $d_{0} / D_{50}$ & $\psi_{\max }$ \\
\hline GB1 & $0.017(0.010)$ & $0.22(0.12)$ & $0.077(0.021)$ & $0.46(0.14)$ & 33 & 2.5 & $2.2 \cdot 10^{3}$ & 110 \\
\hline GB2 & $0.014(0.010)$ & $0.20(0.11)$ & $0.070(0.024)$ & $0.48(0.10)$ & 41 & 2.9 & $2.3 \cdot 10^{3}$ & 175 \\
\hline GB3 & $0.013(0.009)$ & $0.19(0.08)$ & $0.067(0.023)$ & $0.70(0.03)$ & 47 & 3.2 & $2.5 \cdot 10^{3}$ & 117 \\
\hline GM1 & $0.013(0.005)$ & $0.20(0.09)$ & $0.066(0.017)$ & $0.50(0.16)$ & 41 & 2.6 & $2.1 \cdot 10^{3}$ & 167 \\
\hline GM2 & $0.016(0.013)$ & $0.23(0.14)$ & $0.068(0.023)$ & $0.33(0.04)$ & 34 & 2.3 & $2.1 \cdot 10^{3}$ & 138 \\
\hline M1 & $0.020(0.013)$ & $0.25(0.16)$ & $0.078(0.022)$ & $0.81(-)$ & 34 & 2.7 & $2.7 \cdot 10^{3}$ & 116 \\
\hline M2 & $0.033(0.027)$ & $0.38(0.24)$ & $0.081(0.027)$ & $0.62(-)$ & 24 & 2.0 & $3.1 \cdot 10^{3}$ & 170 \\
\hline M3 & $0.032(0.023)$ & $0.57(0.17)$ & $0.053(0.020)$ & $(-)$ & 26 & 1.4 & $3.3 \cdot 10^{3}$ & 212 \\
\hline
\end{tabular}

1053

1054 
1055 Table 4. Measured reference concentrations and depth-averaged sediment diffusivities over the near1056 bed mixing layer.

\begin{tabular}{|l|c|c|c|}
\hline Condition & $C_{\text {ref }}\left(\mathrm{kg} / \mathrm{m}^{3}\right)$ & $\begin{array}{c}\text { Measured } \epsilon \text { over } \\
\zeta / \Delta_{\mathrm{r}}=0 \text { to } 3 \\
\left(\cdot 10^{-3} \mathrm{~m}^{2} / \mathrm{s}\right)\end{array}$ & $\begin{array}{c}\text { Measured } \epsilon /\left(U_{0} \Delta_{\mathrm{r}}\right) \\
\text { over } \zeta / \Delta_{\mathrm{r}}=0 \text { to 3 }\end{array}$ \\
\hline M1 & $9.6 \pm 2.9$ & $0.62 \pm 0.05$ & $0.064 \pm 0.005$ \\
\hline M2 & $5.9 \pm 1.2$ & $0.88 \pm 0.15$ & $0.045 \pm 0.008$ \\
\hline M3 & $4.1 \pm 0.8$ & $0.88 \pm 0.23$ & $0.043 \pm 0.011$ \\
\hline GB1 & $9.1 \pm 5.0$ & $0.61 \pm 0.08$ & $0.082 \pm 0.011$ \\
\hline GB2 & $11.8 \pm 6.1$ & $0.49 \pm 0.05$ & $0.077 \pm 0.007$ \\
\hline GB3 & $8.7 \pm 2.8$ & $0.51 \pm 0.06$ & $0.084 \pm 0.011$ \\
\hline GM1 & $6.0 \pm 2.0$ & $0.52 \pm 0.09$ & $0.095 \pm 0.017$ \\
\hline GM2 & $5.5 \pm 1.8$ & $0.54 \pm 0.08$ & $0.078 \pm 0.011$ \\
\hline
\end{tabular}

1057

1058

1059 
Figures:

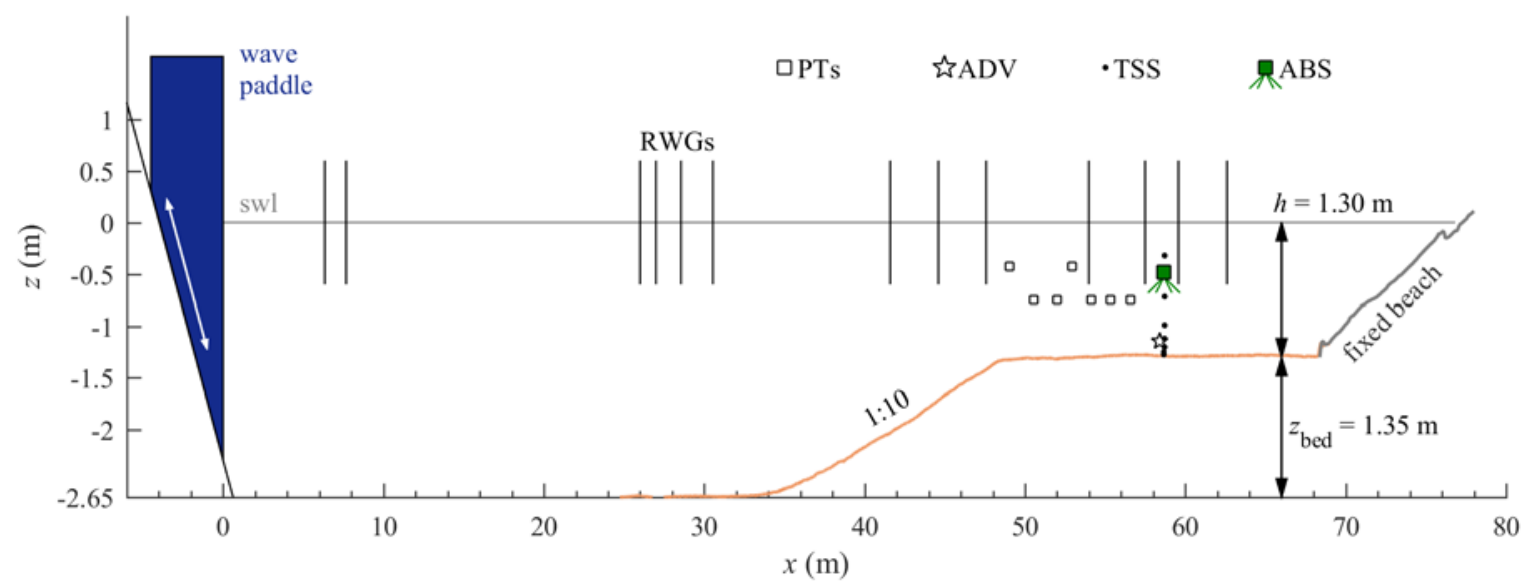

1061

1062

1063

Figure 1. Experimental set-up, including positions of the resistive wave gauges (RWGs), pressure 1064 transducers (PTs), acoustic Doppler velocimeter (ADV), transverse suction system (TSS) nozzles, and acoustic backscatter sensor (ABS).

1065

1066

1067 


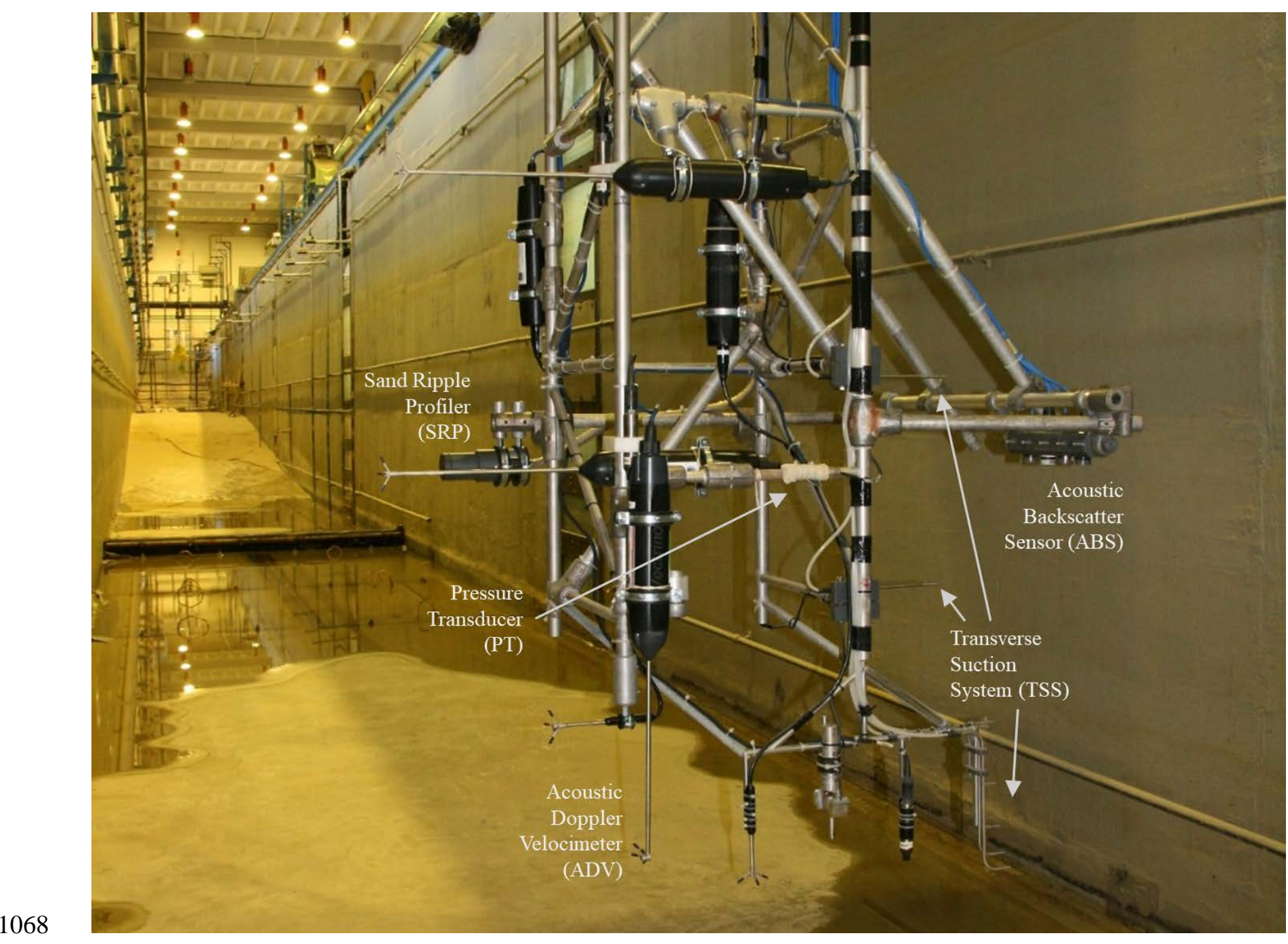

Figure 2. Photograph of instrumentation on the measurement frame. The frame is positioned here in 1071 the deep part of the flume (around $x=15 \mathrm{~m}$ ), the photograph is taken looking towards the test section and beach. 

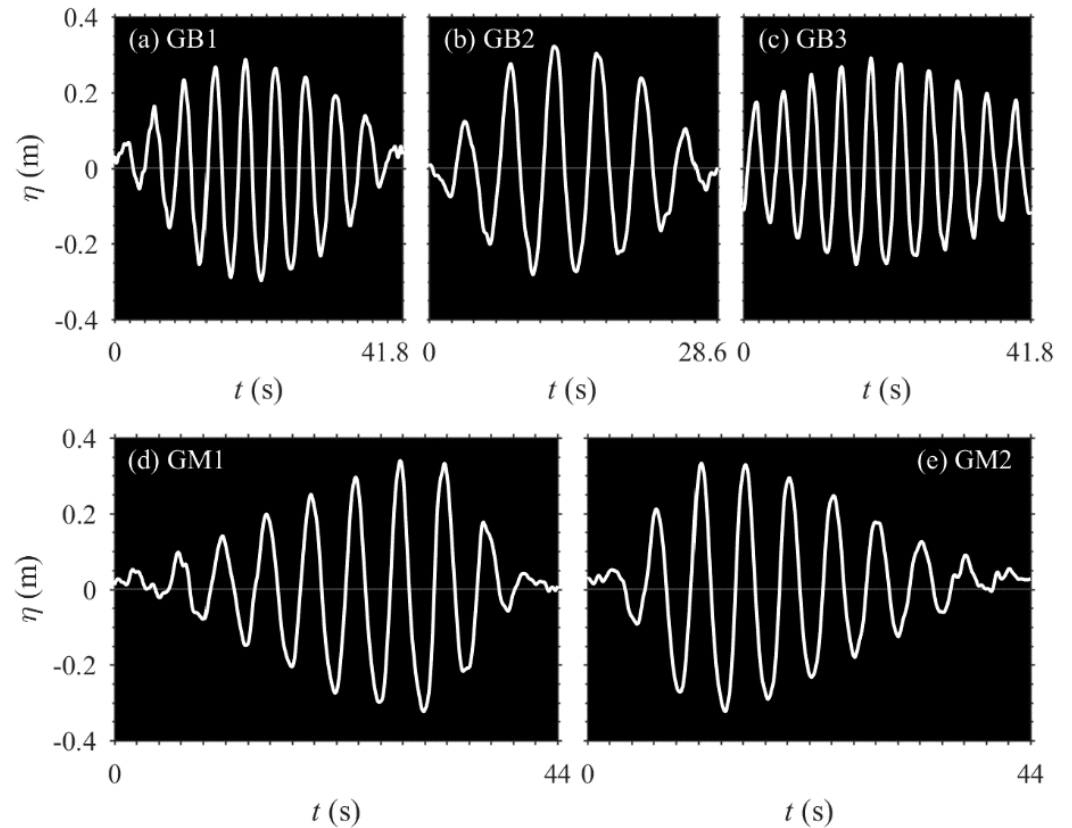

1074

1075 1076

1077

1078

Figure 3. Phase-averaged water surface elevation near the wave paddle for the wave group conditions GB and GM, measured by the RWG at $x=6.3 \mathrm{~m}$. 

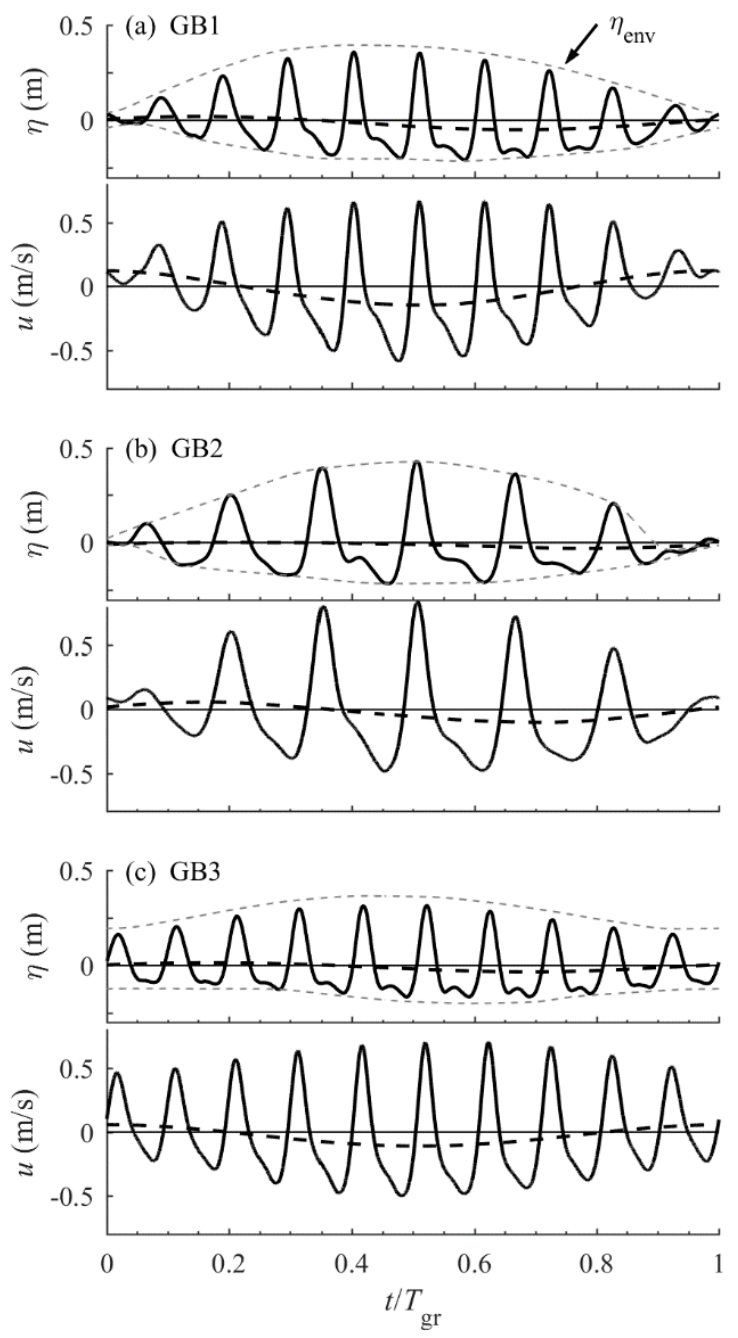

1079

Figure 4. Time series of phase-averaged water elevation $\eta$ and horizontal free-stream velocity $u$ at $\zeta=$ $0.16 \mathrm{~m}$ for the bichromatic groups GB1 (a), GB2 (b) and GB3 (c) (solid lines). Black dashed lines mark low-pass filtered water elevation $\eta_{\mathrm{lf}}$ and velocity $u_{\mathrm{lf}}$. Grey dashed lines indicate wave group envelope $\eta_{\text {env }}$. 

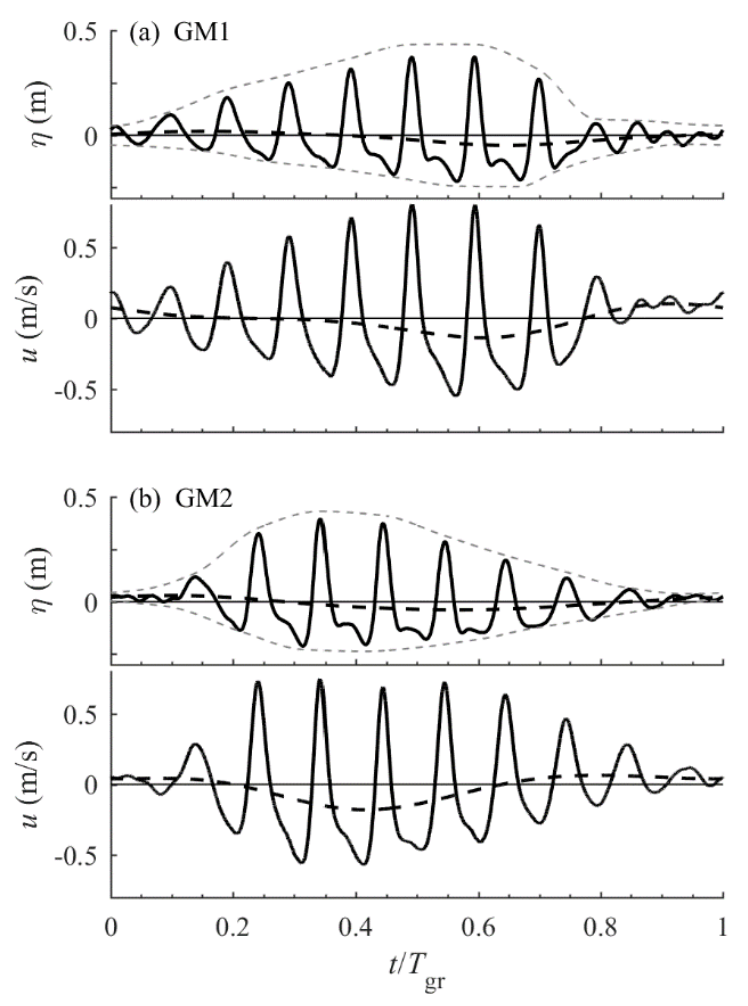

Figure 5. Time series of phase-averaged water elevation $\eta$ and horizontal free-stream velocity $u$ for the sawtooth-modulated groups GM1 (a) and GM2 (b). Black dashed lines mark low-pass filtered water elevation $\eta_{\text {If }}$ and velocity $u_{\text {lf }}$. Grey dashed lines indicate wave group envelope $\eta_{\text {env }}$. 


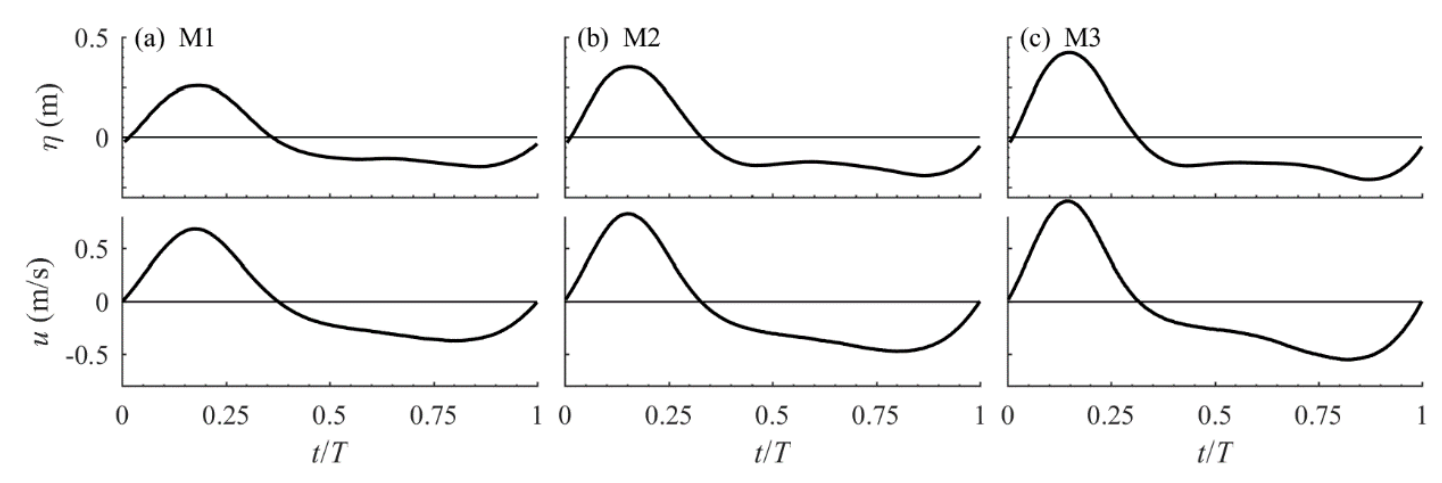

1094

Figure 6. Time series of phase-averaged water elevation $\eta$ (top) and horizontal free-stream velocity $u$ (bottom) for the monochromatic conditions M1 (a), M2 (b) and M3 (c).

1097

1098 


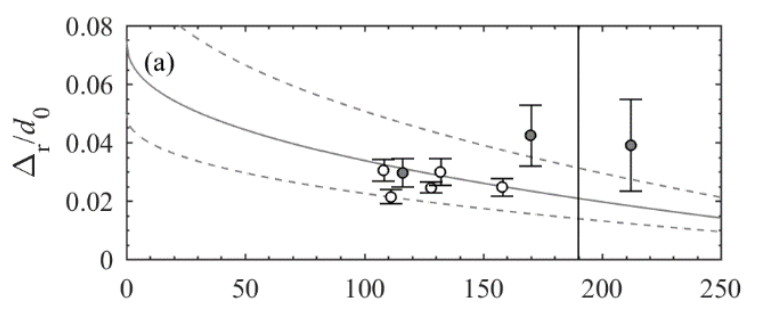

1099

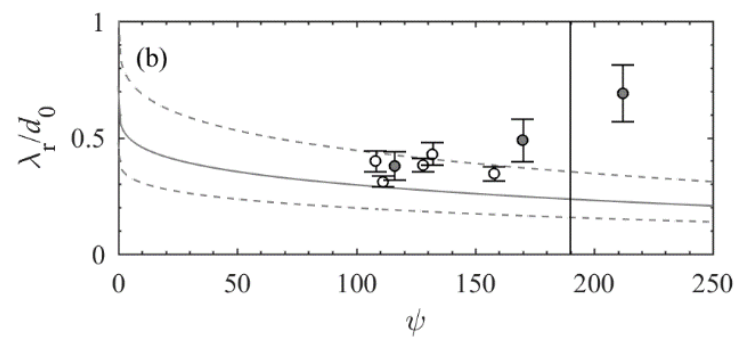

1100

1101

1102

1103

1104

Figure 7. Non-dimensional ripple height (a) and length (b) as function of mobility number.

Measurements are denoted by white (wave groups) and grey (monochromatic waves) markers, the error bars indicate \pm one standard deviation. Solid line indicates empirical prediction by O'Donoghue et al. (2006), dashed lines indicate $\pm 50 \%$. The vertical line marks the range of applicability $(\psi<190)$ of the empirical formula. 

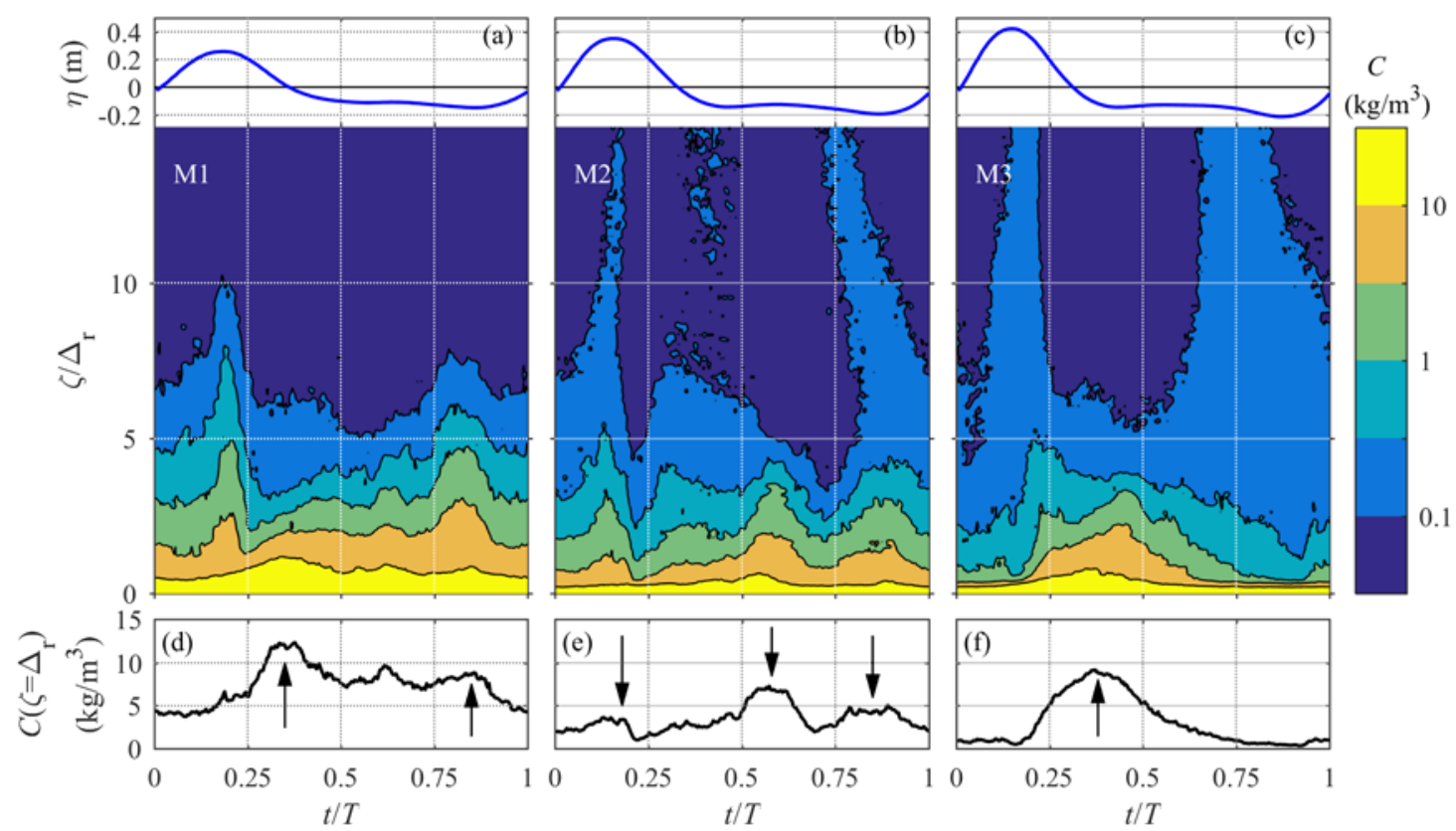

Figure 8. (a-c) Water surface elevation and colour contour plots of the phase-averaged suspended sand concentration field $C(\zeta, t)$ for the monochromatic wave conditions M1 (left), M2 (middle) and M3

(right). Note the logarithmic scale of the colour axis. (b-f) Phase-averaged concentration at $\zeta=\Delta_{\mathrm{r}}$, with arrows indicating the primary pickup events. 

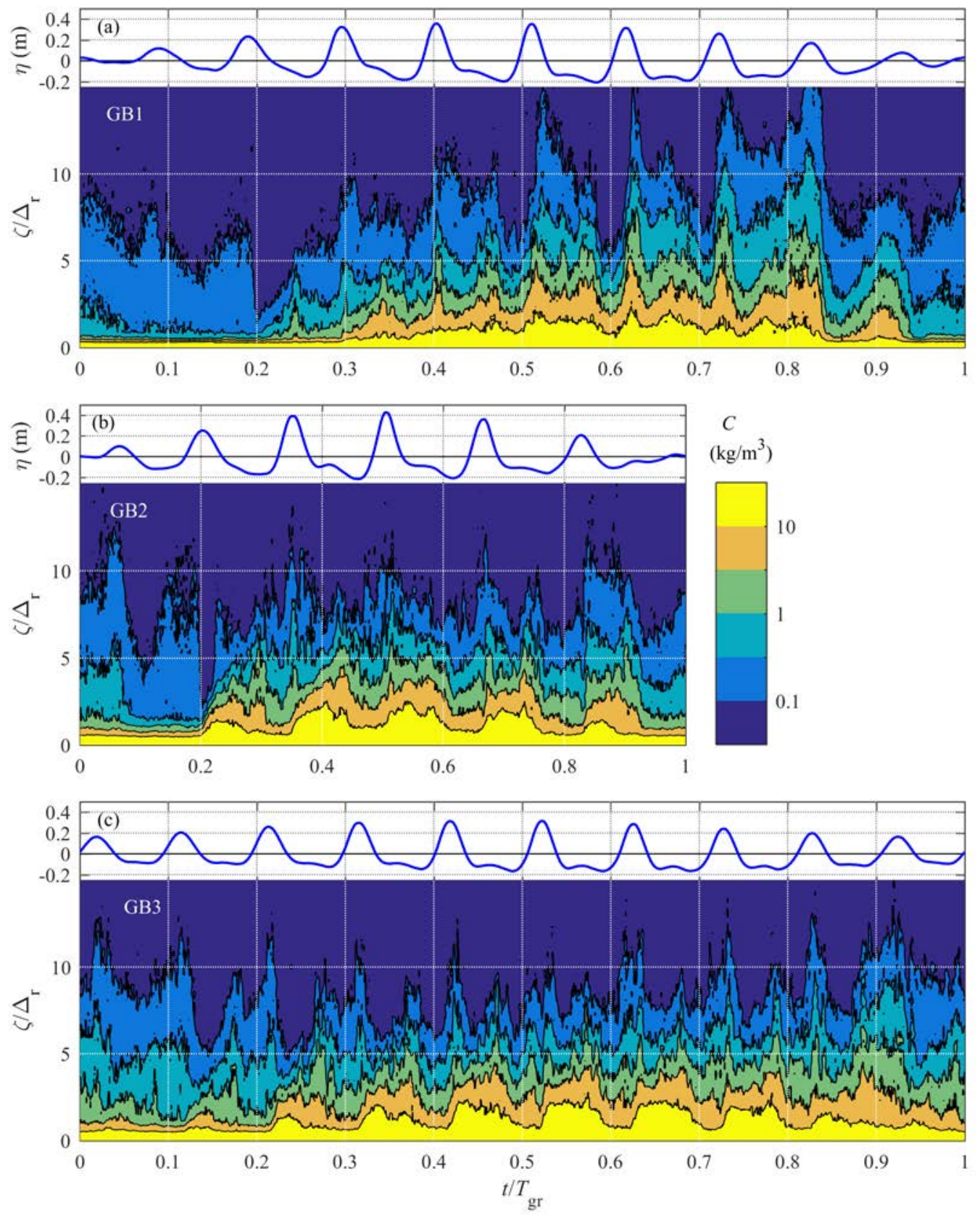

Figure 9. Colour contour plots of the phase-averaged suspended sand concentration field $C(\zeta, t)$ for the bichromatic wave groups GB1 (a), GB2 (b) and GB3 (c). Each panel includes the phase-averaged water surface level $\eta$ (blue solid line) for reference. Note the logarithmic scale of the colour axis. 

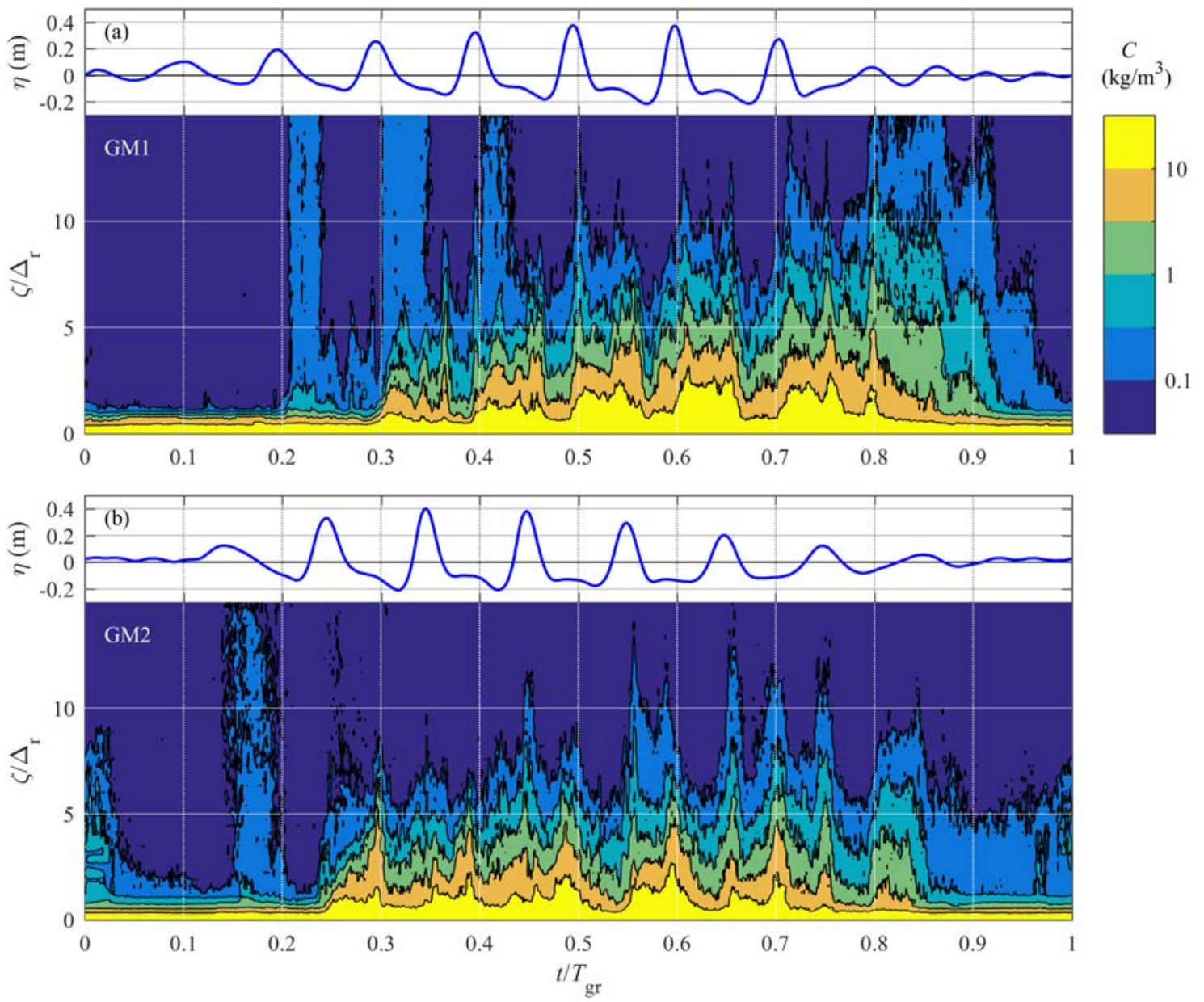

Figure 10. Colour contour plots of the phase-averaged suspended sand concentration field $C(\zeta, t)$ for the sawtooth-modulated wave groups GM1 (a) and GM2 (b). Note the logarithmic scale of the colour axis. 

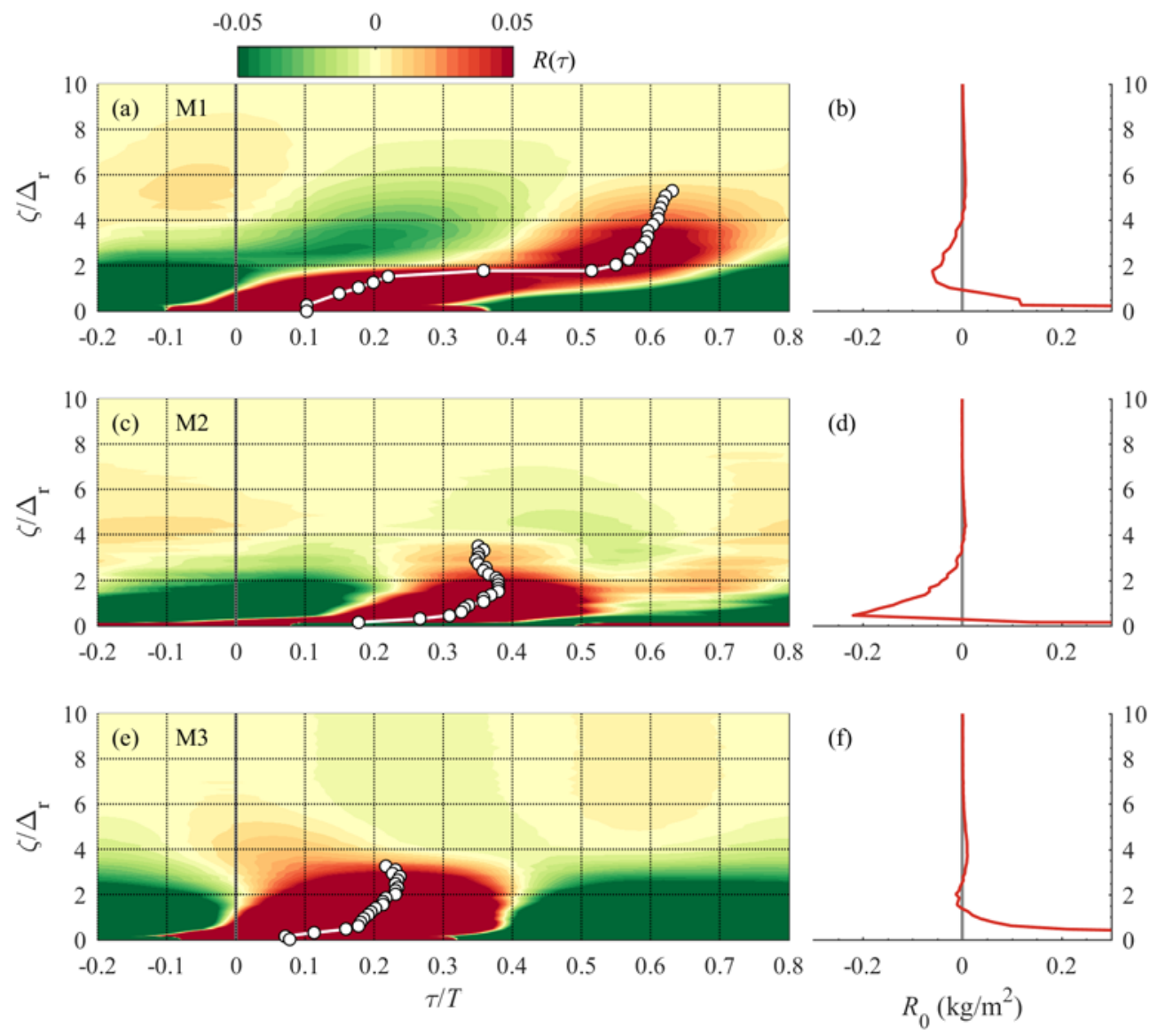

Figure 11. Cross-correlation $R(\zeta, \tau)$ between the water surface level and $C(\zeta, t)$, monochromatic conditions M1 (a,b), M2 (c,d) and M3 (e,f). Left panels: colour contours of $R(\zeta, \tau)$, with the solid white circles indicating the phase lag $\tau_{\max } / T$ between SSC and $\eta$; Right panels: cross-correlation at $\tau=0$. 

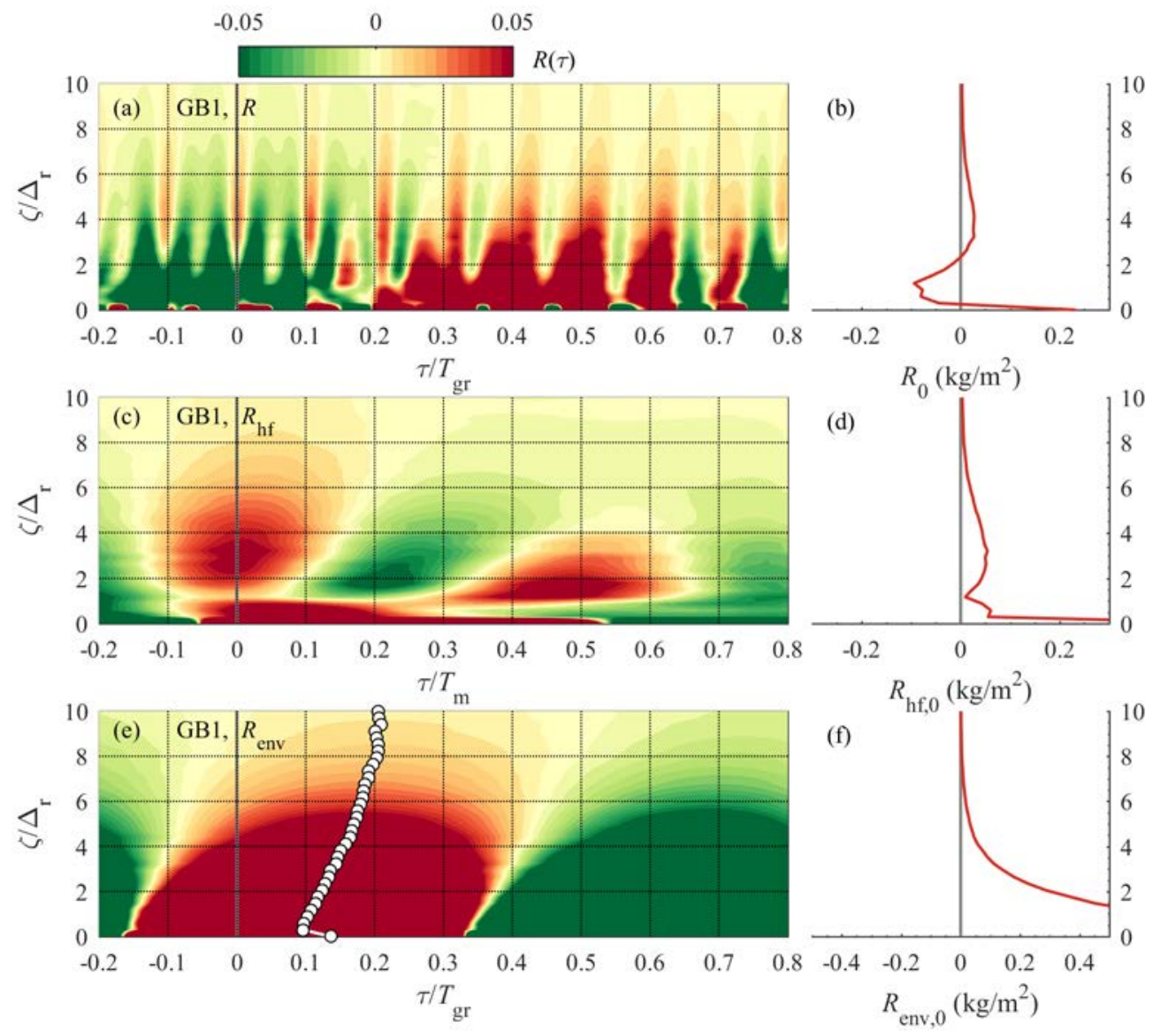

Figure 12. Cross-correlation $R(\zeta, \tau)$ (left) and $R_{0}(\zeta)$ (right) between the water surface elevation and $C(\zeta, t)$ for condition GB1. (a,b) cross-correlation $R$ between $\eta(t)$ and $C(\zeta, t)$; (c,d) cross-correlation $R_{\mathrm{hf}}$ water surface envelope $\eta_{\text {env }}(t)$ and low-pass filtered $C_{\mathrm{lf}}(\zeta, t)$, with solid white circles marking the phase lag distribution. 

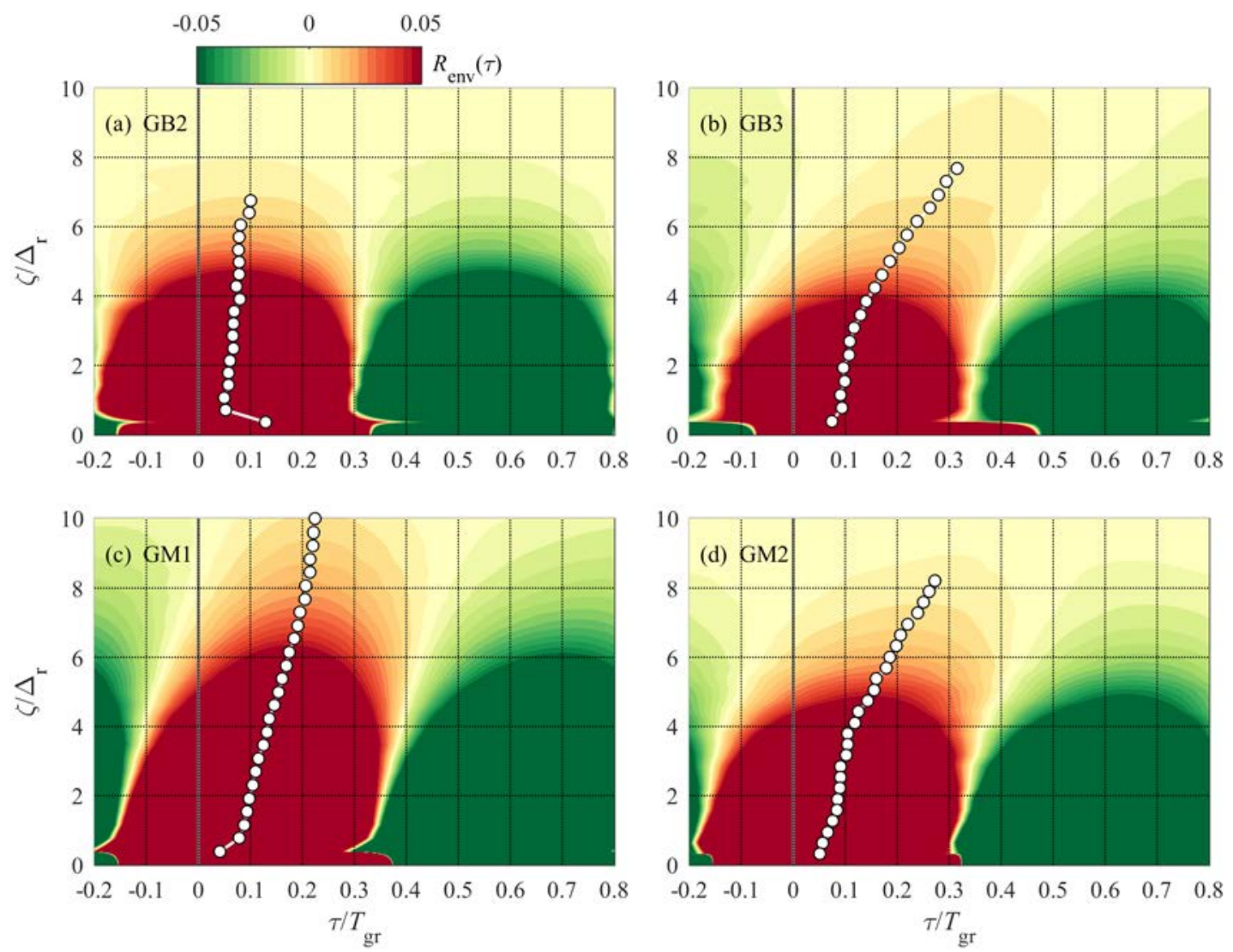

Figure 13. Cross-correlation $R_{\text {env }}$ between the water surface envelope $\eta_{\text {env }}(t)$ and low-pass filtered $C_{\text {lff }}(\zeta$, t) for conditions (a) GB2; (b) GB3; (c) GM1; (d) GM2. White solid circles indicate the phase lag $\tau_{\max }$ between $C_{\text {lf }}$ and $\eta_{\text {env }}$. 

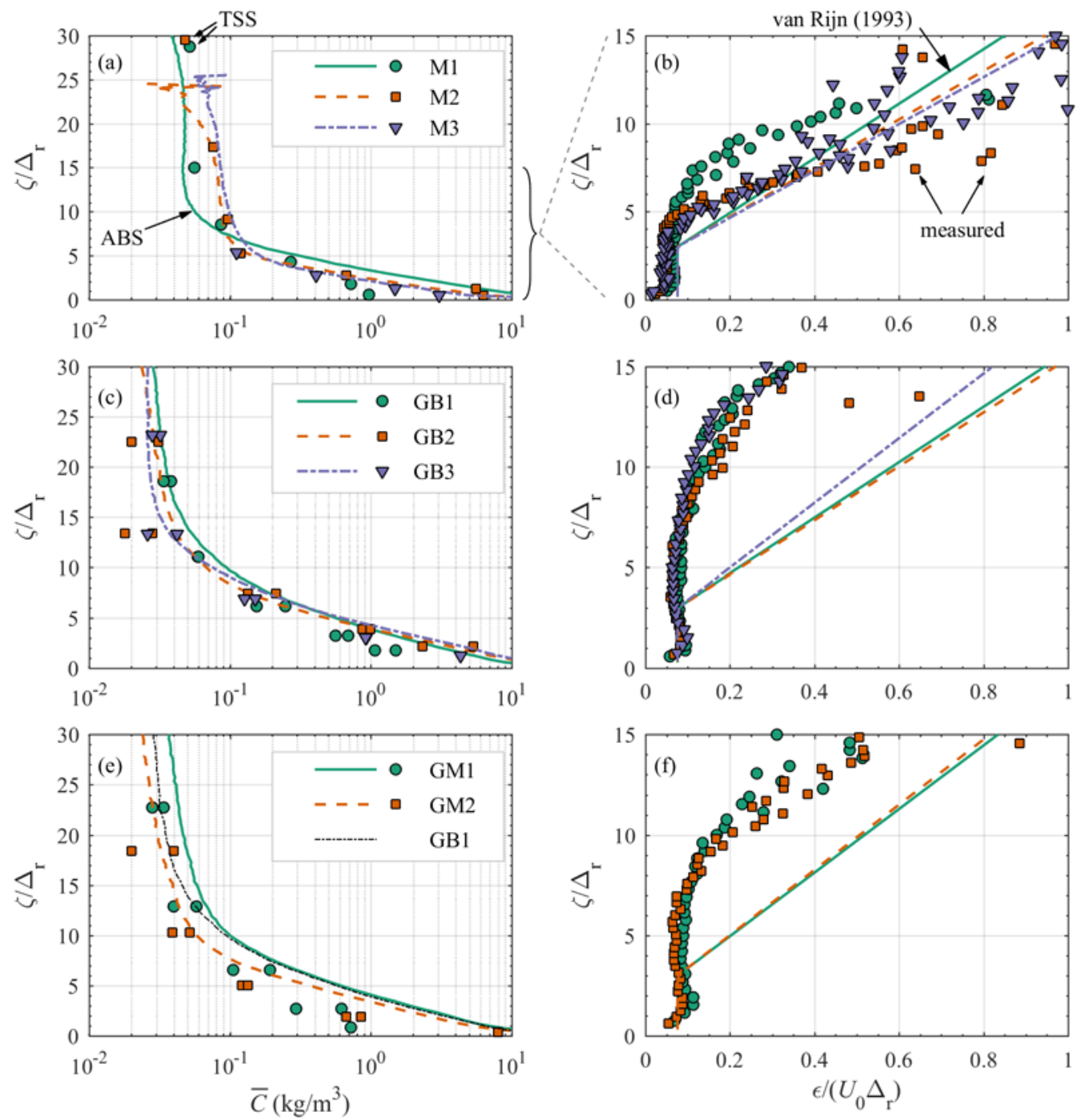

1140 Figure 14. Vertical profiles of the time-averaged concentration $\bar{C}(\zeta)$ (left) and sediment diffusivity $\epsilon(\zeta)$ (right), for wave conditions M1-3 (top), GB1-3 (middle) and GM1-2 (bottom). (a, c, e) $\bar{C}(\zeta$ ) measured with ABS (lines) and TSS (markers) (to facilitate comparisons, panel (e) also includes $\bar{C}(\zeta)$ for GB1); (b, d, f) $\epsilon(\zeta)$ from ABS measurements (markers) and predictions following Equation (12) (lines). 

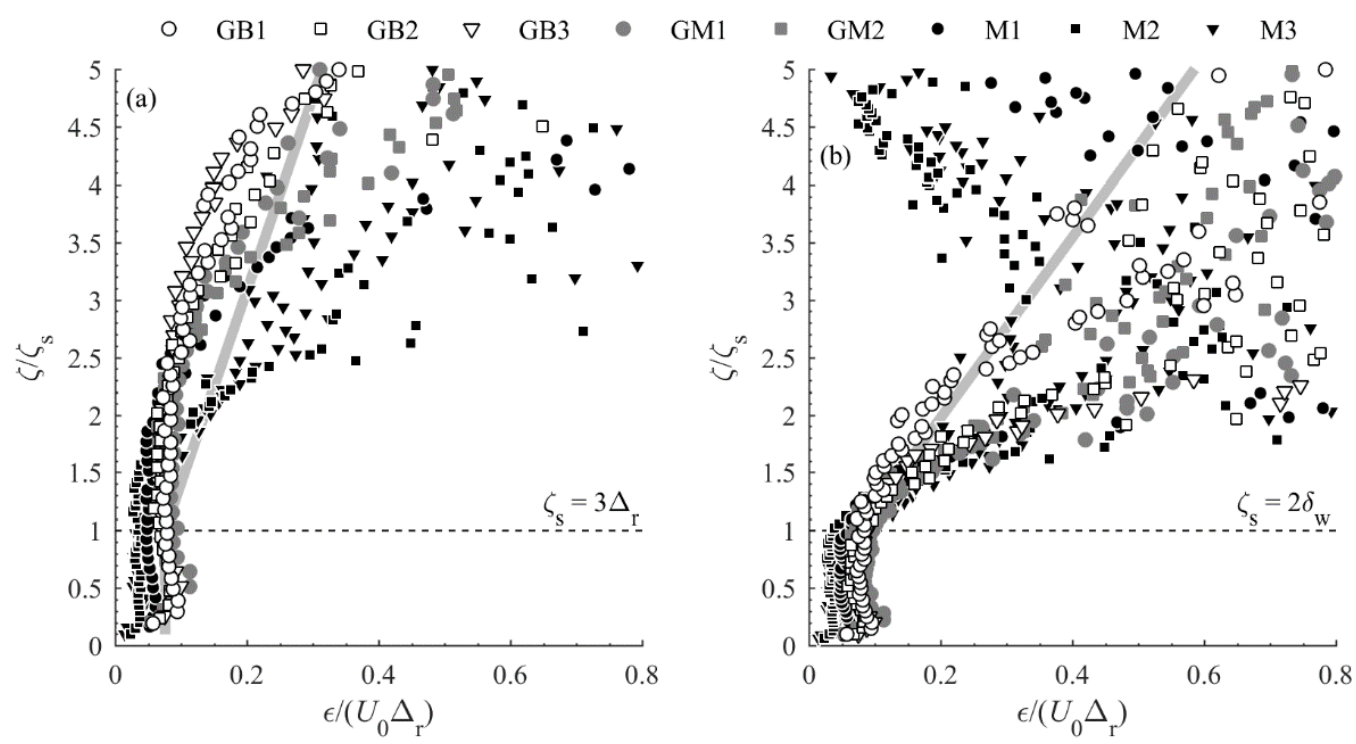

Figure 15. Vertical profiles of the sediment diffusivity using different vertical scaling for the vertical axis: (a) elevations normalized by $\zeta_{\mathrm{s}}=3 \Delta_{\mathrm{r}}$; (b) elevations normalized by $\zeta_{\mathrm{s}}=2 \delta_{\mathrm{w}}$. The symbols represent the measured $\epsilon(\zeta)$ for all wave conditions, the solid line represents the predicted $\epsilon(\zeta)$ following Equation (12) for condition IGB1. 


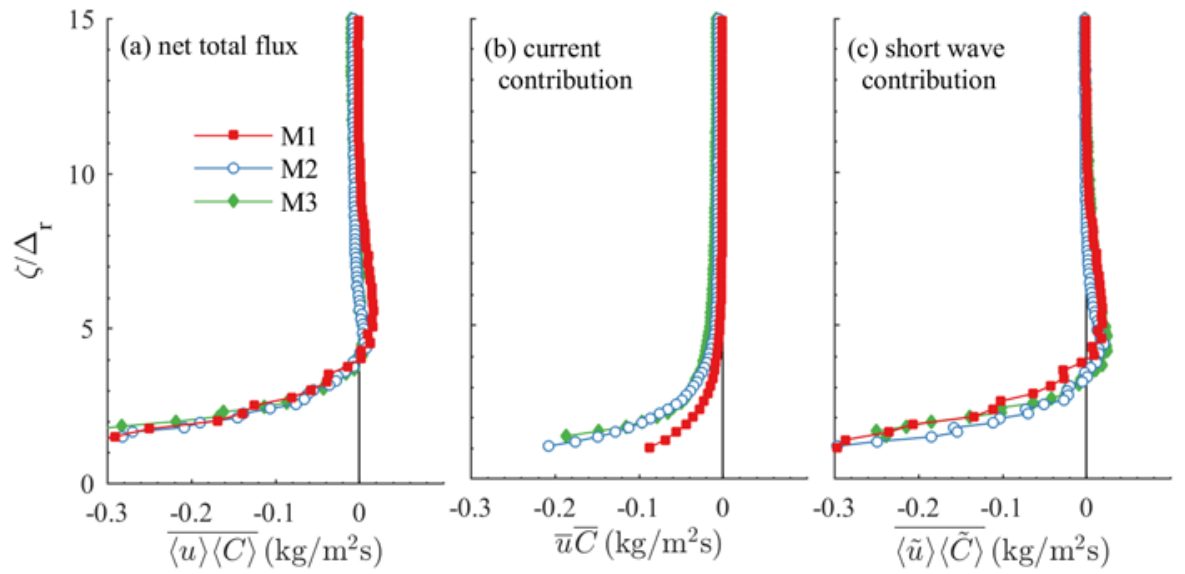

1152 Figure 16. Net horizontal suspended sand flux distributions for the monochromatic conditions. (a) Net total flux; (b) Current-related flux contribution; (c) Short-wave flux contribution. 

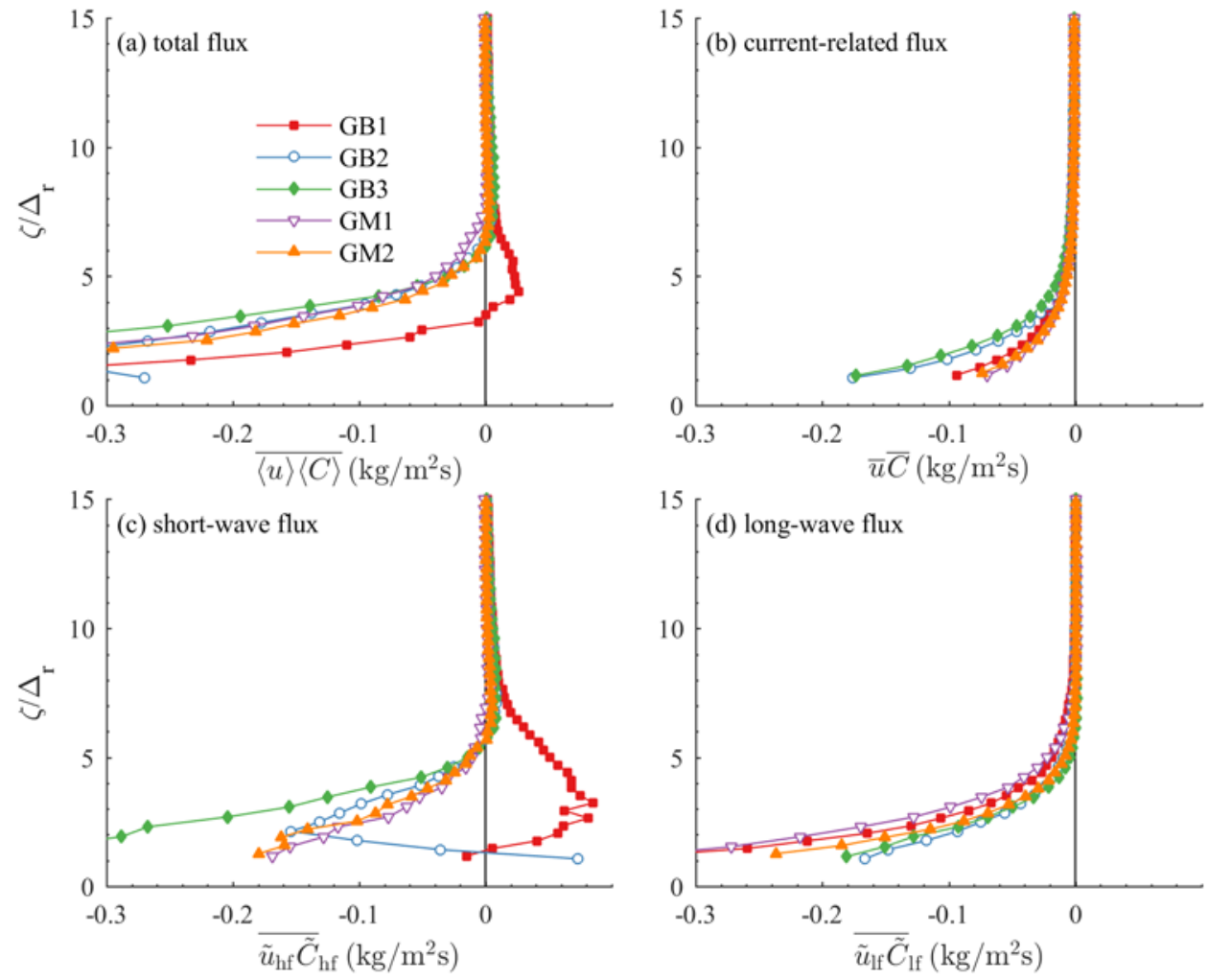

1155

1156

1157

Figure 17. Net horizontal suspended sand flux distributions for the wave group conditions. (a) Net total flux; (b) Current-related flux; (c) Short-wave flux; (d) Long-wave flux. 


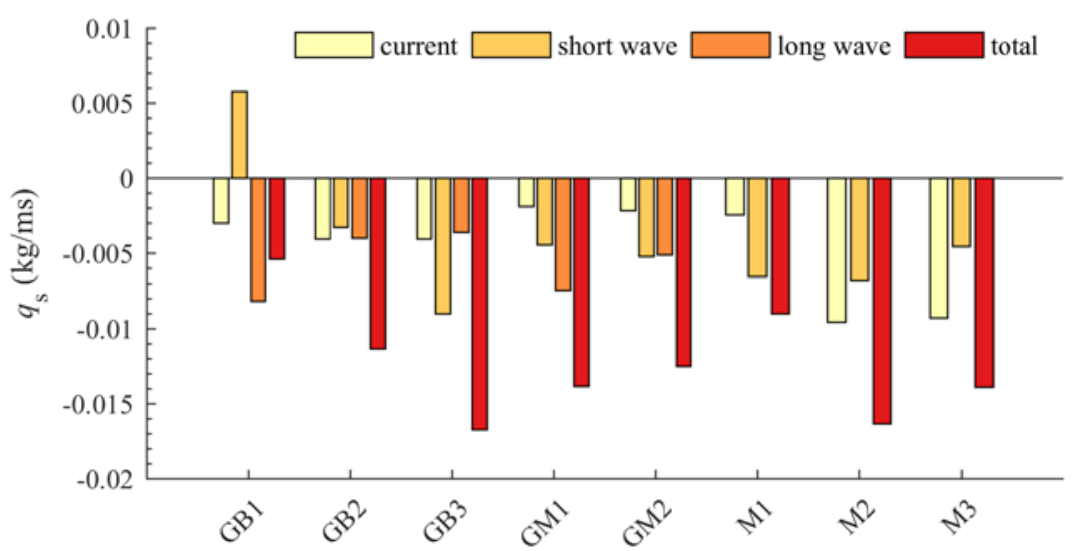

Figure 18. Net depth-integrated suspended transport between $\zeta=\Delta_{\mathrm{r}}$ and $0.50 \mathrm{~m}$, decomposed into 1161 contributions by the current, short waves, and the long wave. Note that there is no long-wave transport 1162 for M1-3. 


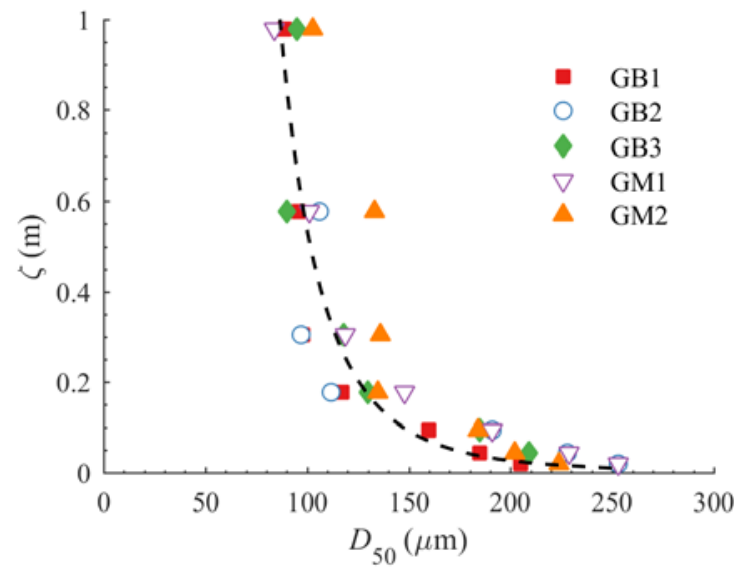

1166

1167

Figure A1. Vertical profiles of the median grain diameter $D_{50}$. Symbols indicate the measured $D_{50}$ of the suction samples, dashed line indicates power-law fit (Equation (A1)).

1168

1169 